Michael W. Busch \& Karin Link

Was macht Agilität mit Macht?

\section{Journal Härr Psychologie}

29. Jahrgang 2021 - Hett 1

Aglle Organisationen

Versuch einer kritischen Bestandsautnahme

-

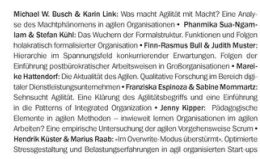

Journal für Psychologie

29. Jahrgang, Nr. 1, 2021, Seite 9-38

Psychosozial-Verlag

DOI: 10.30820/0942-2285-2021-1-9 


\section{Impressum}

Journal für Psychologie

Theorie - Forschung - Praxis

Zeitschrift der Neuen Gesellschaft

für Psychologie (NGfP)

www.journal-fuer-psychologie.de

ISSN (Online-Ausgabe): 2198-6959

ISSN (Print-Ausgabe): 0942-2285

29. Jahrgang, 2021, Heft 1

Herausgegeben von Andrea Birbaumer und Ralph Sichler

https://doi.org/10.30820/0942-2285-2021-1

ISBN der Print-Ausgabe: 978-3-8379-8354-8

ViSdP

Die HerausgeberInnen; bei namentlich

gekennzeichneten Beiträgen die AutorInnen.

Namentlich gekennzeichnete Beiträge stellen nicht in jedem Fall eine Meinungsäußerung der

HerausgeberInnen, der Redaktion oder des Verlages dar.

\section{Herausgebende}

Mag. Andrea Birbaumer, Wien · Dr. Martin Dege, Berlin · Dr. Peter Mattes, Berlin/Wien · Prof. Dr.

Günter Mey, Magdeburg-Stendal/Berlin · Dr. Aglaja

Przyborski, Wien • Paul Sebastian Ruppel, Bochum .

Univ.-Doz. Dr. Ralph Sichler, Wiener Neustadt · Dr.

Anna Sieben, Bochum/Jülich · Prof. Dr. Thomas

Slunecko, Wien

\section{Wissenschaftlicher Beirat}

Prof. Dr. Molly Andrews · Prof. Dr. Thea Bauriedl . Prof. Dr. Jarg Bergold · Prof. Dr. Klaus-Jürgen Bruder • Prof.

Dr. Stefan Busse · Prof. Dr. Tanja Eiselen • Prof. Dr. Jörg Frommer · Prof. Dr. Heiner Keupp · Prof. Dr. Carlos Kölbl · Prof. Dr. Helmut E. Lück · PD Dr. Günter Rexilius · Prof. Dr. Dr. h.c. Wolff-Michael Roth • Prof. Dr. Christina Schachtner - Prof. Dr. Rudolf Schmitt . Prof. Dr. Ernst Schraube • Prof. Dr. Margrit Schreier . Prof. Dr. Hans-Jürgen Seel · Dr. Michael Sonntag . Prof. Dr. Hank Stam · Dr. Irene Strasser, Klagenfurt · Prof. Dr. Dr. Wolfgang Tress • Prof. Dr. Jaan Valsiner - Dr. Barbara Zielke · Prof. Dr. Dr. Günter Zurhorst

\section{Erscheinen}

Halbjährlich als Organ der Neuen Gesellschaft für Psychologie (NGfP) als Open-Access-Publikation und parallel als Print-Ausgabe.

\author{
Verlag \\ Psychosozial-Verlag \\ Walltorstraße 10 \\ D-35390 Gießen \\ info@psychosozial-verlag.de \\ www.psychosozial-verlag.de
}

\section{Abonnentenbetreuung}

aboservice@psychosozial-verlag.de

\section{Bezug}

Jahresabonnement 49,90€ (zzgl. Versand)

Einzelheft 29,90€ (zzgl. Versand)

Studierende erhalten gegen Nachweis 25\% Rabatt, Mitglieder der NGfP erhalten 30\% Rabatt auf den Preis des Jahresabonnements.

Das Abonnement verlängert sich um jeweils ein Jahr, sofern nicht eine Abbestellung bis acht Wochen vor Beendigung des Bezugszeitraums erfolgt.

\section{Anzeigen}

Anfragen richten Sie bitte an den Verlag:

anzeigen@psychosozial-verlag.de

DieZeitschrift Journal für Psychologie wird regelmäßig in der Publikationsdatenbank PSYNDEX des Leibniz-Institut für Psychologie/Leibniz Institute for Psychology (ZPID) erfasst.

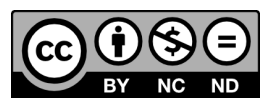

Die Beiträge dieser Zeitschrift sind unter der Attribution-NonCommercial-NoDerivatives 4.0 International Lizenz (CC BY-NC-ND 4.0) lizensiert. Diese Lizenz erlaubt die private Nutzung und unveränderte Weitergabe, verbietet jedoch die Bearbeitung und kommerzielle Nutzung. Weitere Informationen finden Sie unter: creativecommons.org/ licenses/by-nc-nd/4.0/ 


\title{
Was macht Agilität mit Macht?
}

\section{Eine Analyse des Machtphänomens in agilen Organisationen}

\author{
Michael W. Busch \& Karin Link \\ Journal für Psychologie, 29(1), 9-38 \\ https://doi.org/10.30820/0942-2285-2021-1-9 \\ CC BY-NC-ND 4.0 \\ www.journal-fuer-psychologie.de
}

\section{Zusammenfassung}

Agilität prägt seit einigen Jahren die organisationale Beratungspraxis und wird nicht selten als das unternehmerische $\gg$ Allheilmittel « für mehr Flexibilität, Mitarbeiterorientierung etc. gehandelt. Das Thema Macht - als ein zentrales Phänomen organisationaler Beeinflussungsprozesse - wird dabei kaum diskutiert. Aufbauend auf einem interdisziplinären Zugang widmen wir uns in diesem Beitrag der Frage, welche Rolle das Phänomen der Macht in agilen Organisationen einnimmt. Unser besonderer Fokus liegt hierbei auf Teams, da diese in agilen Konzepten eine zentrale Rolle einnehmen. Wir zeigen, dass in agilen Organisationen primär auf die Kraft der Überzeugung durch informale Macht und demokratische Willensbildung gesetzt wird, während in der klassisch-hierarchisch strukturierten Organisation der formalen Macht größeres Gewicht als der informalen Macht zukommt. Doch auch in agilen Organisationen sind Regeln und hierarchieähnliche $\gg$ Letztentscheidungen « erforderlich. Wie genau sich diese im Einzelfall auszugestalten haben, ist durch die jeweiligen kontextuellen Gegebenheiten und Restriktionen zu klären.

Schlüsselwörter: Agilität, Macht, Teams

\section{Summary}

What does agility do with power?

An analysis of the phenomenon of power in agile organizations

Organizational agility has emerged as a central topic in organizational consulting practice recently. Very often agile concepts are seen as the entrepreneurial » panacea « for more flexibility, employee orientation, etc. However, the topic of power in agile contexts is hardly discussed in contemporary literature. Based on an interdisciplinary approach, this article discusses the question of what role the phenomenon of power plays in agile organizations. 
We thereby focus on teams, as teams play a central role in agile concepts. We show that while in classically hierarchical organizations formal power is more important than informal power, agile organizations primarily rely on the power of persuasion through informal power and democratic decision-making. Still, formal rules and hierarchy-like »final decisions « play an essential role also in agile contexts. How these formal rules and »final decisions « could best be shaped relies on the respective contextual conditions and restrictions.

Keywords: Organizational agility, power, teams

Wo die Liebe herrscht, da gibt es keinen Machtwillen.

Carl Gustav Jung

\section{$1 \quad$ Einleitung und Überblick}

Das Thema Agilität prägt seit einigen Jahren die organisationale Beratungspraxis. Agilität wird geradezu als unternehmerisches »Allheilmittel « angepriesen, um den vielschichtigen Herausforderungen der sogenannten VUCA-Welt zu begegnen. Als zentrale Konzepte werden hierbei die aus der Softwarentwicklung stammende Methode Scrum (Glogler 2016), Holacracy (Robertson 2016) und das schon ältere partizipative Entscheidungsmodell der Soziokratie (Strauch und Reijmer 2018) diskutiert. Diese Konzepte gehen zum Teil auf frühere Ansätze aus der Selbstorganisationsforschung zurück (Probst 1992) und finden, angestoßen durch das $2001 \mathrm{im}$ Internet veröffentlichte Agile Manifest, zunehmend Verbreitung im betrieblichen Alltag (Porschen-Hueck et al. 2020). Zum Thema Agilität gibt es bislang jedoch kaum empirisch belastbare Erkenntnisse; auch fehlt es an theoretisch fundierender und kritischer Forschung (Grundei und Kaehler 2018, 428). Unser Beitrag hat nicht die Aufarbeitung empirischer Studien zum Ziel. Vielmehr soll dieser Problemaufriss zur theoretischen Fundierung und Systematisierung beitragen, indem ein in der Agilitätsliteratur zentraler, jedoch zu sehr vernachlässigter, ja verschleierter Aspekt thematisiert wird, nämlich die Frage, welche Rolle das Phänomen der Macht in agilen Organisationen einnimmt.

Hierbei wird ein interdisziplinärer Zugang gewählt, der soziologische, politikwissenschaftliche, betriebswirtschaftliche und (sozial-)psychologische Erkenntnisse berücksichtigt. Dadurch soll ein ganzheitliches und realistisches Verständnis des Machtphänomens in agilen Organisationen - und hier insbesondere in Teams - 
gewonnen werden, denn dieses ist unseres Erachtens zu komplex, als dass es einzelwissenschaftlich gelöst werden könnte. Im Zentrum unserer Analyse steht die Unterscheidung zwischen formaler und informaler Macht. Agile Organisationskonzepte wie zum Beispiel Holokratie versuchen, die formale Entscheidungsmacht von höheren auf niedrigere Hierarchiestufen zu verlagern; an die Stelle der hierarchischen Kontrolle tritt die Klärung von Problemen im Rahmen von Rollenbefugnissen und demokratischen Aushandlungs- und Einflussprozessen. Gerade letztere bieten informalen Machtphänomenen eine Bühne, auf der lenkende Einflussgrößen - individuelle Stärken, Sympathie oder Beziehungen - wirksam werden. Indem diese Einflussformen analysiert, theoretisch verortet und kritisch reflektiert werden, soll ein differenzierteres Bild von Agilität im Lichte der Machtproblematik gezeichnet werden. Dabei kommen sowohl erkennbare Stärken als auch mögliche Schwächen dieses Konzepts zur Sprache. Mit den drei genannten Kräften liegt ein konzeptioneller Bezugsrahmen vor, der als Orientierungshilfe und Analyseinstrument für Wissenschaft und Praxis dienen kann.

Die Vorstellung, die Machtfrage hätte sich »erledigt «, sobald Hierarchien mit klar definierten Regeln und Zuständigkeiten abgeschafft und durch selbstorganisierende, flexible und demokratische Strukturen ersetzt werden, greift unseres Erachtens deutlich zu kurz. Macht prägt stets zwischenmenschliche Beziehungen; es gibt im Grunde keine machtfreien Zonen im Sozialen. Das Machtphänomen ist auch in agilen Strukturen präsent. Es tritt hier vermehrt in einer anderen, weicheren und weniger durchschaubaren Gestalt in Erscheinung. Wir folgen in unserem Beitrag der Grundannahme des österreichisch-französischen Soziologen Erhard Friedberg, nach der jede ernst zu nehmende Organisationsanalyse Macht ins Zentrum ihrer Überlegungen zu stellen hat (Friedberg 1992, 40).

\section{Macht in Organisationen}

Macht ist ein zentrales Phänomen in Organisationen. Für diesen Beitrag orientieren wir uns an der klassischen Definition von Max Weber (1972, 28), wonach Macht verstanden wird als »jede Chance, innerhalb einer sozialen Beziehung den eigenen Willen auch gegen Widerstreben durchzusetzen, gleichviel, worauf diese Chance beruht «. Diese $\gg$ Chance «, das heißt die Möglichkeit, dass eine Person Einfluss auf eine andere Person(-engruppe) ausüben kann bzw. diese die Einflussausübung zulässt, wird in der Literatur mit unterschiedlichen Begrifflichkeiten diskutiert und tritt in der Praxis in unterschiedlichen Formen auf. Das folgende Kapitel widmet sich daher der Gegenüberstellung wesentlicher Begriffspaare, die mit dem Phänomen Macht assoziiert werden. 


\subsection{Formen der Macht}

\subsubsection{Künstliche und natürliche Macht}

Eine für das allgemeine Verständnis des Machtphänomens zentrale Unterscheidung hat Thomas Hobbes in seinem 1651 erschienenen Buch Leviathan vorgenommen, das als Klassiker der modernen Staatstheorie gilt.

»Es gibt aber eine natürliche und eine künstliche Macht. Die natürliche Macht gründet sich auf außerordentliche Vorzüge des Körpers oder des Geistes, zum Beispiel auf Stärke, Gestalt, Klugheit, Geschicklichkeit, Beredsamkeit, Freigiebigkeit und Adel. Die künstliche Macht aber umfasst die Mittel und Werkzeuge, seine Macht zu erhöhen, in sich; sie mögen übrigens durch jene ersteren, oder durch Zufall erlangt sein, wie: Reichtum, Achtung, Freunde und die unmerkliche Einwirkung Gottes, welche gewöhnlich das Glück genannt wird $\ll($ Hobbes 1996, 69).

In Organisationen zeigt sich künstliche Macht in der zugewiesenen hierarchischen Position, die bestimmte Machtmittel zur Verfügung stellt (z. B. Ressourcen) und Formen der Machtausübung gewährt (z. B. das Recht, Meetings einzuberufen oder Beurteilungen vorzunehmen). Natürliche Macht tritt beispielsweise im Charisma oder allgemein in der Überzeugungskraft einer Person in Erscheinung. Wie Hobbes bereits erkannt hat, steht künstliche Macht, wenn sie auf der Basis natürlicher Macht, also außerordentlicher Vorzüge, erlangt wurde, auf einem solideren Fundament.

\subsubsection{Formale und informale Macht}

Diese Unterscheidung ist auch für Organisationen relevant, um die dort ablaufenden Einflussprozesse zu begreifen, die notwendig sind, damit Ziele überhaupt erreicht werden können. Erfolgt der Einfluss »von oben «, so wird tendenziell von formaler bzw. hierarchischer (Führungs-) Macht gesprochen; erfolgt er hingegen »von unten «, so wird tendenziell von informaler bzw. heterarchischer (Führungs-)Macht gesprochen (Luhmann 1999, 283). Tendenziell deshalb, weil auch Führungskräfte mit formaler Rollenmacht über informale Macht verfügen und umgekehrt Mitarbeiter*innen ohne Führungsfunktion eine gewisse formale Macht besitzen können (z. B. als Betriebsratsmitglied oder Gleichstellungsbeauftragte). Beide Formen der Beeinflussung von Akteuren sind in der Realität verschränkt, können sich gegenseitig ergänzen, verstärken, aber auch blockieren. Informale Macht kann Defizite formaler Macht ausgleichen, so wie formale Machtträger informal erkannte Änderungsnotwendigkeiten torpedieren und sabotieren können (Reimann 2017, 20,23). Während formale Macht auf künstlich errichteten Machtquel- 
len fußt (etwa in der Möglichkeit, Mitarbeiter*innen zu bestrafen oder zu belohnen, einzustellen oder zu entlassen), gründet informale Macht auf natürlichen, das heißt der Person eigenen bzw. der Person von der Umwelt zuerkannten Machtquellen (z. B. Expertise, Reife, Integrität). Der formale Status wird temporär (z. B. Projektleitung) oder dauerhaft (z. B. Abteilungsleitung) verliehen, den informalen Status muss sich der Betreffende erarbeiten (z. B. durch vertrauensfördernde Maßnahmen [Malik 2006, 140-156], inhaltliche Überzeugungskraft, wertvolle Inputs, durch verlässliches, wertschätzendes Verhalten, Arbeitseinsatz und Fleiß), letztlich wird ihm dieser aber durch die Gruppe eingeräumt bzw. attribuiert (Keltner 2016, 74; Mitchell 1995, 848). Der informale Status ist seiner Natur nach entsprechend dynamischer und brüchiger. Bei ausbleibender Bewährung, das heißt, wenn der oder die Betreffende keinen erkennbaren Nutzen mehr für die Gruppe erbringt oder gravierende Vertrauensbrüche begangen hat, kann der Status wieder entzogen werden. Um ihn zu erhalten oder zu erhöhen, sind also fortlaufende Anstrengungen durch den Inhaber erforderlich. Im Vergleich dazu ist der formale Status stabiler, speziell dann, wenn Rückhalt von höherer Ebene gegeben ist. Der formale Status zeigt sich nicht nur in äußerlich erkennbaren Unterscheidungsmerkmalen (z. B. häufig größeres Büro oder höheres Gehalt), sondern auch in eher unauffälligen Machtindikatoren (z. B. Foto im Geschäftsbericht, Sprachstil) (Schmidt et al. 2020, 147-149).

\subsubsection{Kompetenz und Autorität}

In der älteren Forschung wurden mit Kompetenz und Autorität zwei Begriffe verwendet, die in eine ähnliche Richtung weisen. Der Kompetenzbegriff folgt hier nicht dem modernen Verständnis einer durch sichtbare Erfolge gekennzeichneten Aufgabenbewältigung (von Krogh und Roos 1995, 62), sondern dem klassischen Verständnis organisational zugeteilter Rechte oder Befugnisse (aber auch Pflichten und Verantwortungen auf der Kehrseite). Hierzu zählen

$>$ Entscheidungsbefugnisse (Rechte auf Treffen von Entscheidungen),

$>$ Anordnungs-bzw. Weisungsbefugnisse (Rechte auf Bestimmung des Verhaltens anderer Stellen, d. h. Personalführungsverantwortung),

$>$ Verpflichtungsbefugnisse (Rechte auf Verpflichtung der Unternehmung gegenüber Dritten, z. B. Abschluss von Kaufverträgen),

$>$ Verfügungsbefugnisse (Rechte auf Verfügung über Sachen und Werte, z. B. Budgetverantwortung),

$>$ Vertretungsbefugnisse (Rechte auf Vertretung abwesender Personen) und

> Informationsbefugnisse (Rechte auf Information) (Ulrich 1969, 852-853, teilweise ergänzt). Letztere zeigen sich heutzutage besonders in Zugriffsrechten auf unternehmensinterne Daten und die Kontrolle über Kommunikationswege (allgemein Rifkin 2000). 
Kompetenzen hängen eng mit der Autorität des Stellenträgers zusammen, jedoch ist beides nicht dasselbe.

»In personeller Betrachtungsweise stellt die Kompetenz die formale Seite der Autorität dar. Die mit Autorität gemeinte Fähigkeit eines Vorgesetzten, das Verhalten anderer Menschen in der Unternehmung in einer von ihm gewünschten Richtung zu beeinflussen, hängt stark - aber nicht ausschließlich - von den ihm offiziell zugebilligten Kompetenzen ab. Werden einem Chef von der Unternehmungsleitung nicht genügende Kompetenzen zugeteilt, so leidet darunter seine Autorität bei den Untergebenen und seine Fähigkeit, als Menschenführer wirksam zu werden« (Ulrich 1969, 855).

Besitzen Vorgesetzte nur formale Kompetenzen (Amtsautorität), aber wenig persönliche Autorität, so werden sie aus Sicht der Geführten meist als schwache oder unfähige Führungskräfte wahrgenommen. Darunter kann die allgemeine Arbeitsmoral der Belegschaft leiden; verdeckte Formen des passiven Widerstands oder der Leistungszurückhaltung sind möglich. Oft müssen solche Führungskräfte dann auf repressive Formen der Machtausübung, auf gezielte Machtdemonstrationen wie dem Statuieren eines Exempels, zurückgreifen, um sich überhaupt Geltung zu verschaffen. Die vermehrte Verwendung von Maßregelungen führt jedoch zu einer schleichenden Machtentwertung (von Ameln und Kramer 2012, 194).

Der Autoritätsbegriff reicht also wesentlich weiter als der Kompetenzbegriff (zur ideengeschichtlichen Verortung des Autoritätsbegriffs Sichler 2006, 230-242 sowie aktuell Baumann-Habersack 2017). Autorität gründet in einer allgemeinen oder spezifischen Überlegenheit, die es dem Autoritätsträger erlaubt, das Handeln eines anderen vollständig oder teilweise zu beeinflussen, wobei »die Betroffenen die Überlegenheit und die Einflussnahme freiwillig anerkennen. Das grenzt die Autorität von anderen Einflüssen, die etwa auf Zwang oder Manipulierung beruhen, ab « (Landwehrmann 1969, 269). Autorität vereint demnach sowohl formale als auch informale Machtelemente und betrifft einerseits die Person als Mensch, andererseits die Person als Rolleninhaber einer Führungsposition innerhalb der organisationalen Hierarchie.

\subsubsection{Herrschaft und Macht}

Das Spannungsverhältnis zwischen formaler, institutionalisierter Macht und informaler, personalisierter Macht hat auch Max Weber in seiner Unterscheidung von Herrschaft und Macht herausgestrichen. Diese soll hier als letzte und vermutlich bekannteste Darstellung des Problems zwischen künstlich geschaffener und natürlich gewachsener, zugeschriebener Macht aufgeführt werden. Zusätzlich zu der bekannten, bereits vorgestellten Definition Webers von Macht (2.) heißt es bei ihm an anderer Stelle, bei 
Macht handle es sich um ein » soziologisch amorphes « Phänomen. Hinter dieser etwas kryptischen Formulierung steckt ein einfacher Gedanke: Macht ist in der Gesellschaft etwas Allgegenwärtiges. Es gibt keine sozialen Beziehungen ( $\mathrm{ob}$ im betrieblichen, familiären, therapeutischen, politischen oder schulischen Kontext), in denen Macht keine Rolle spielt. Sie ist stets präsent. Die Suche nach einem machtfreien Raum im zwischenmenschlichen Zusammenleben ist daher von vornherein vergeblich. Kommt Macht nicht im offiziellen Gewand der Hierarchie einher, so tritt sie durch die Hintertür im Gewand der Informalität ein. »Offensichtliche werden dann nur durch subtilere Formen der Über- und Unterordnung ersetzt « (Busch und von der Oelsnitz 2018, 223; vgl. auch Reimann 2017,20). Bertrand Russell $(2001,10)$ ging daher so weit, zu behaupten, dass Macht ein für die Sozialwissenschaften ebenso fundamentales Konzept darstellt wie Energie für die Physik. Die Quellen, aus denen sich Macht speisen kann, sind dabei vielfältiger Natur (z. B. Intelligenz, Wissen und Können, Ausstrahlung) (Popitz 1992 und Han 2005 zu grundlegenden Analysen des Machtphänomens). Ihr Wirkungsgrad hängt nicht zuletzt davon ab, inwieweit sie aus Sicht des Beherrschten als persönlich bedeutsam oder auch nützlich eingestuft werden. Die Motivationstheorie nennt dies Valenz (Anziehungskraft, Attraktivität). Wofür jemand empfänglich ist, variiert allerdings von Person zu Person. Einfluss kann jemand über einen anderen nur dann ausüben, wenn er dessen $\gg N e r v \ll$ trifft, das heißt, die emotionale Verbindung (Rapport) zu ihm herzustellen vermag.

Beruht Macht allein auf der Position innerhalb einer organisationalen Hierarchie, so spricht Weber von Herrschaft, die er im Vergleich zu Macht wesentlich enger, nämlich als »die Chance, für einen Befehl bestimmten Inhalts bei angebbaren Personen Gehorsam zu finden «, definiert (Weber 1972,28). Die erlaubten Inhalte werden durch die zugewiesenen Befugnisse festgelegt, der angebbare Kreis von Personen durch die Anzahl der untergeordneten Stellen. Außerhalb dieses Kreises besitzt der Herrschende vielleicht Ansehen, aber keine echte Macht. Gemäß der Unterscheidung zwischen Herrschaft und Macht kann ein Teammitglied über ein anderes Mitglied mehr Einfluss haben als die formal ernannte Teamführung (z. B. aufgrund seiner Freundlichkeit oder Hilfsbereitschaft). Ein Mensch muss demnach nicht immer über eine hohe Position in der Organisation oder Gesellschaft verfügen, um mächtig zu sein. Ein berühmtes Beispiel hierfür ist Nelson Mandela. Mandela avancierte bereits während seiner Gefangenschaft trotz oder gerade wegen seiner ohnmächtigen Situation zu einer » mächtigen « Identifikations- und Symbolfigur. Macht muss also nicht Herrschaft inkludieren, aber Herrschaft inkludiert stets ein gewisses Maß an (Sanktions-)Macht (nach dem Prinzip »Ober sticht Unter «). Tabelle 1 fasst die bisherigen Erkenntnisse zusammen.

Mit der Frage der unterschiedlichen Möglichkeiten und Grundlagen zur Durchsetzung von Macht beschäftigt sich das folgende Kapitel. 


\begin{tabular}{|c|c|}
\hline \multicolumn{2}{|c|}{ Chance der Einflussausübung auf Mitmenschen } \\
\hline Künstliche Macht & Natürliche Macht \\
\hline $\begin{array}{l}\text { Mittel und Werkzeuge zur Machterhö- } \\
\text { hung }\end{array}$ & $\begin{array}{l}\text { Außerordentliche Vorzüge des Körpers } \\
\text { oder des Geistes }\end{array}$ \\
\hline Formale Macht & Informale Macht \\
\hline $\begin{array}{l}\text { Hierarchische Positionsmacht (personen- } \\
\text { unabhängig; erzwungene Gefolgschaft) }\end{array}$ & $\begin{array}{l}\text { Individueller Einfluss (abhängig von der } \\
\text { Person, ihrer Leistung bzw. der Zuschrei- } \\
\text { bung durch die Umwelt; freiwillige Ge- } \\
\text { folgschaft) }\end{array}$ \\
\hline Kompetenz & Autorität \\
\hline $\begin{array}{l}\text { Organisational zugeteilte Befugnisse } \\
\text { (z. B. Entscheidungs- und Weisungsbe- } \\
\text { fugnisse) }\end{array}$ & $\begin{array}{l}\text { Allgemeine oder spezifische Überlegen- } \\
\text { heit, durch die der Träger Anerkennung } \\
\text { und Respekt genießt (»Standing") }\end{array}$ \\
\hline Herrschaft & Macht \\
\hline $\begin{array}{l}\text { Die Chance, für einen Befehl bestimmten } \\
\text { Inhalts bei angebbaren Personen Gehor- } \\
\text { sam zu finden }\end{array}$ & $\begin{array}{l}\text { Jede Chance, innerhalb einer sozialen Be- } \\
\text { ziehung den eigenen Willen auch gegen } \\
\text { Widerstreben durchzusetzen, gleichviel, } \\
\text { worauf diese Chance beruht }\end{array}$ \\
\hline ste Machtkonzentration bzw & $\begin{array}{l}\text { as größte Einflussvermögen liegt vor, } \\
\text { tgrundlagen in sich vereint. }\end{array}$ \\
\hline
\end{tabular}

Tab. 1: Chance der Einflussausübung auf Mitmenschen

\subsection{Formen zur Durchsetzung von Macht: Hard Power und Soft Power}

Eine hierarchische Stellung mit Formalmacht verleiht im Vergleich zur rein informalen Macht weit umfassendere Möglichkeiten, Menschen zu beeinflussen, um sie in die gewünschte Richtung zu lenken. Die Führungsperson, die nicht nur ihre Stellung innehat, sondern zudem Autorität bei den Mitarbeiter*innen besitzt, ist noch mächtiger und kann sowohl auf »Hard Power « als auch auf »Soft Power « zurückgreifen. Einem informalen Machtträger steht dagegen allein die Möglichkeit der weichen Beeinflussung zur Verfügung (z. B. Überzeugung durch Wissen, Bildung von Koalitionen). Hard Power umfasst eher tangible, Soft Power eher intangible Ressourcen. Der Umgang mit Informationen (Zurückhaltung, Glättung, Verteilung, Interpretation) nimmt hierbei eine Zwischenstellung ein - als Machtmittel »von oben « oder Gerücht »von unten «. Hard Power und Soft Power repräsentieren zwei gegensätzliche Pole der Machtdurchsetzung:

$>$ Zwang versus Freiwilligkeit,

$>$ Angst (Schreckensbild) versus Zuneigung (Vorbild), 
$>$ Kalkül (Gehorsam erkaufen) versus Identifikation (Gehorsam gewinnen),

$>$ (An-)Drohung bis hin zur physischen Gewaltanwendung versus psychologische Vereinnahmung durch subtilere, verständnisfördernde Formen der Beeinflussung.

Der Politikwissenschaftler Joseph Nye hat das Begriffspaar Soft Power und Hard Power am Ende des Kalten Krieges ursprünglich für den Kontext internationaler Beziehungen eingeführt (Nye 1990; Wilson 2008, 114). Militärische Gewalt und materielle Ressourcen (»Wer zahlt, schafft an«) stehen hierbei exemplarisch für den Bereich der Hard power. Mit diesen kann der Träger Zwang ausüben: Drohen, Bestrafen (von Sanktionen bis zum Einsatz von Gewalt) und »Kaufen «. Berüchtigt ist Mao Zedongs Ausspruch »Alle politische Macht kommt aus den Gewehrläufen «. Demgegenüber besteht Soft power in immateriellen Ressourcen. Mit diesen kann der Träger den Beeinflussten überzeugen. Hierzu zählen etwa technisches Know-how, Hilfeleistungen, Beteiligung an Diskussionszirkeln (» prozessuale Eingebundenheit «; Patchen 1974), Persönlichkeit, visionäre Kraft, kreative Ideen sowie kulturelle Normen, Werte und Überzeugungen bzw. Ideologien und Glaubenssysteme. In einer kalten, technizistischen Sprache ließe sich von »cultural engineering « sprechen - bei ethischem Missbrauch, zum Beispiel in Sekten, werden Begriffe wie »Gehirnwäsche«, »Indoktrination $\ll$, $\gg$ Mental Programming « oder $\gg$ Mind Control « verwendet. Hard und Soft Power sind demnach als wertneutrale Phänomene aufzufassen, die sowohl nutzbringend gebraucht als auch zerstörerisch missbraucht werden können. Hard Power kann »gut « sein (z. B. Sanktionen gegen einen gewalttätigen Regelbrecher) genauso wie Soft Power »schlecht « sein kann (z.B. durch den Einsatz manipulativer Methoden).

Mit weichen Ressourcen kann der Träger den Beeinflussten »kooptieren«, das heißt, zu einem Gleichgesinnten machen. Ähnlich Denkende werden am Ende zu ähnlich Handelnden. Der Wille des Beeinflussten gleicht sich dem Willen des Einflussnehmers an. Repressive Formen der Machtausübung und -demonstration sind bei überzeugten Mitstreitern wenn überhaupt nur noch in Ausnahmefällen erforderlich. »Parents of teenagers have long known that if they have shaped their child's beliefs and preferences, their power will be greater and more enduring then if they rely only on active control « (Nye 1990, 166). Weich ist somit auch hart. Zwar ist die Umsetzung und der Erwerb für den Träger weicher Ressourcen im Vergleich zu harten Ressourcen mühsam und zeitintensiv, doch ist die Erfolgswirksamkeit beim Beeinflussten dafür umso nachhaltiger und effektiver. Konformität, das heißt Gehorsam gegenüber den Anweisungen, erfolgt dann aus innerer Überzeugung, weil die durch den Anweisenden vertretene Grundeinstellung geteilt wird. Die sozialpsychologische Forschung spricht in dem Zusammenhang von Einstellungskonformität (im Gegensatz zur rein äußerlich praktizierten Anpassungskonformität) (Fischer und Wiswede 2009, 613-614). 
Einstellungskonforme Menschen kommen aufgrund ihrer hohen Identifikation mit dem Herrscher bzw. den herrschenden Ideen überhaupt nicht auf den Gedanken, Widerspruch gegen Anordnungen an den Tag zu legen oder Dinge infrage zu stellen. Anpassungskonforme Menschen hingegen trauen sich aus Angst vor Vergeltung nicht, Zweifel zu äußern, obwohl sie womöglich starke innere Vorbehalte hegen. Ihre Anpassung ist rein äußerlich - der klassische Vertreter des Mitläufers (Busch 2014, 141).

In Organisationen wird Hard Power als Androhung und Einsatz von Zwangsmitteln nur selten in Anwendung gebracht. Die Beteiligten sind sich der Existenz dieser Ultima Ratio gleichwohl bewusst. Entsprechend ist die Selbstdisziplinierung der Organisationsmitglieder eine wesentliche Grundlage organisationaler Macht. Diese sorgt dafür, dass den kollektiven Erwartungen (Ziele, Stellenbeschreibungen, Kommunikationswege) und den Entscheidungen der Vorgesetzten in der Regel ohne manifeste Widerstände Folge geleistet wird. Sie bildet eine weitgehend verinnerlichte Richtgröße des individuellen Verhaltens und stabilisiert auf eine unscheinbare Weise die bestehenden Herrschaftsverhältnisse.

Die Unterscheidung in Hard und Soft Power wird in Tabelle 2 als ordnendes Konzept benutzt, um die in der Literatur am häufigsten diskutierten Typologien von Machtquellen zu strukturieren (Pfeffer 1992, 69-186; Sandner 1993, 229-235; Miebach 2012, 73-92; Lang 2014, 186-189 und Morgan 2008, 228-270).

Aufbauend auf den dargelegten Erkenntnissen erfolgt nun eine Einordnung des Machtphänomens in agile Organisationen. Spielen hier klassische Formen der hierarchischen Macht keine Rolle mehr oder treten sie anders, zum Beispiel in Form von Beziehungsnetzwerken oder Sprechzeiten in Meetings, in Erscheinung? Sollte hier überhaupt noch von Macht gesprochen werden oder sind bewusst neue, weichere Formulierungen wie Einflussnahme (Influencing) zu wählen? Woraus speist sich diese Einflussnahme - allein aus der Kraft der überzeugenderen Idee? Und mithilfe welcher theoretischen Erklärungsansätze kann eine Annäherung an das Phänomen der wechselseitigen und zielorientierten Beeinflussung in agilen Organisationen erfolgen?

\section{Macht in agilen Organisationen}

Die Gretchenfrage ist nicht nur in traditionellen, sondern auch in agilen Organisationen die Frage nach der Macht, denn die Machtstruktur einer Organisation ist letztlich der entscheidende Bestimmungsfaktor der Organisationsstruktur (Kieser und Kubicek 1992, 445). Agile Organisationskonzepte wie zum Beispiel Holokratie verankern Entscheidungsbefugnisse und damit Macht in Rollen, Teams oder Kreisen, die in ihrem jeweiligen Verantwortungsbereich schnell, flexibel und situativ gut angepasst handeln sollen. Diese Aspekte der formalen Machtübertragung auf die Mitarbeiter*innen bzw. 


\begin{tabular}{|c|c|c|}
\hline Typologie nach ... & Hard Power & Soft Power \\
\hline French/Raven (1959) & $\begin{array}{l}\text { Belohnungsmacht } \\
\text { Bestrafungsmacht } \\
\text { Positionsmacht }\end{array}$ & $\begin{array}{l}\text { Informationsmacht } \\
\text { Identifikationsmacht } \\
\text { Expertenmacht }\end{array}$ \\
\hline Etzioni (1961) & $\begin{array}{l}\text { Physische Macht } \\
\text { (Zwang, Androhung oder } \\
\text { Gebrauch von Gewalt) } \\
\text { Utilitaristische Macht } \\
\text { (Geld/Anreiz, ökonomi- } \\
\text { sche Sanktionen) }\end{array}$ & $\begin{array}{l}\text { Überredungsmacht } \\
\text { (Symbole, Ideen, Lob) }\end{array}$ \\
\hline Galbraith (1983) & $\begin{array}{l}\text { Repressive Macht (Unter- } \\
\text { drückung) } \\
\text { Kompensatorische Macht } \\
\text { (Leistung und Gegenleis- } \\
\text { tung) }\end{array}$ & $\begin{array}{l}\text { Konditionierende Macht } \\
\text { (mentale bzw. kulturelle } \\
\text { Beeinflussung durch Nor- } \\
\text { men, Werte und Informa- } \\
\text { tionen) }\end{array}$ \\
\hline Bourdieu (1983) & $\begin{array}{l}\text { Ökonomisches Kapital } \\
\text { (finanzielle und materiel- } \\
\text { le Ressourcen wie Geld, Ei- } \\
\text { gentum, Besitz) }\end{array}$ & $\begin{array}{l}\text { Kulturelles Kapital (Wis- } \\
\text { sen, Bildung, Habitus, } \\
\text { Prestige) } \\
\text { Soziales Kapital (formel- } \\
\text { les und informelles Bezie- } \\
\text { hungsnetzwerk, Zugehö- } \\
\text { rigkeit zu einer Gruppe) }\end{array}$ \\
\hline Popitz (1992) & $\begin{array}{l}\text { (Verletzende) Aktions- } \\
\text { macht } \\
\text { (Gewalt, Pranger, Mob- } \\
\text { bing) } \\
\text { Instrumentelle Macht } \\
\text { (Drohungen oder Verspre- } \\
\text { chungen, positive oder } \\
\text { negative Sanktionen) }\end{array}$ & $\begin{array}{l}\text { Autoritative Macht } \\
\text { (Autorität als Maßstab: An- } \\
\text { erkennung führt bei Ab- } \\
\text { hängigen zur Steigerung } \\
\text { des Selbstwertgefühls; } \\
\text { verändert Einstellungen } \\
\text { und Normen) } \\
\text { Datensetzende Macht } \\
\text { (Schaffung technischer Ar- } \\
\text { tefakte wie Architektur } \\
\text { und IT, die Menschen un- } \\
\text { terschwellig beeinflussen) }\end{array}$ \\
\hline
\end{tabular}

Tab. 2: Nach Hard und Soft Power strukturierte Machttypologien

die Bildung verhaltenslenkender Erwartungen und Regeln sollen hier nicht im Zentrum stehen. Unser Fokus liegt vielmehr auf all jenen personengebundenen Formen der Macht, die subtiler und weniger sichtbar wirksam werden.

Denn auch wenn in agilen Organisationen häufig von der Abschaffung, zumindest aber von der radikalen Verflachung hierarchischer Strukturen die Rede ist, gehen wir unserer bisherigen Argumentation folgend - nicht davon aus, dass sich Macht in sozialen Beziehungen einfach ausschalten lässt. 
Sie tritt dann in anderer Form auf, folgt aber auch hier dem Prinzip von Überund Unterordnung: Wer kann sich gegen wen durchsetzen und warum? Selbst im partizipativen Entscheidungsmodell der Soziokratie, in dem Entscheidungen nach dem Konsentprinzip erfolgen und erst dann vollzogen werden, wenn niemand mehr Einwände vorzubringen hat, müssen neben alltäglichen Entscheidungen auch strategisch wichtige Entscheidungen getroffen werden (z.B. Ressourcenvergabe, Erfassung der Arbeitszeit, Arbeitsorganisation, Bestimmung der strategischen Geschäftsfelder). Auf Basis welcher Entscheidungskriterien dies geschieht (z. B. ökonomisch, ökologisch, wertebasiert, empirisch) bzw. wie diese Kriterien gewichtet werden sollen, hängt am Ende aber doch wieder von Einflussverhältnissen, also dem Faktor Macht, ab.

Es sprechen zweifellos stichhaltige Argumente für formale, hierarchische Macht: Sie hat eine höhere Durchschlagskraft, weist eindeutige Zuständigkeiten auf und sorgt für Rollensicherheit, kann Entscheidungen klar und rasch herbeiführen, Fehler transparent rückverfolgen, Konflikte durch ein Machtwort lösen und braucht auf Emotionen und persönliche Befindlichkeiten der Beteiligten streng genommen nur dann einzugehen, wenn die Arbeitsergebnisse zu wünschen übrig lassen (aber auch in diesem Fall werden nicht selten genug nur noch » härtere Bandagen « angelegt, um die gewünschten Ergebnisse $\gg$ herbeizuzwingen «). Informaler Macht hingegen kann vorgehalten werden, dass sie zu schwerfällig ist und im Hinblick auf die Verantwortungszurechnung undurchschaubarer wird. Sie kann zu einer übertriebenen »Psychologisierung « und »Dauerpolitisierung « von sozialen Beziehungen führen. Informale Strukturen »sind potenziell anfälliger für Intriganten und Seilschaften und können von Lautsprechern oder Menschenfängern dominiert werden « (Reimann 2017, 21). Wenn Entscheidungen nurmehr durch das bessere Argument oder die geschicktere Art der Überzeugung und Koalitionsbildung herbeigeführt werden können, dann kommt Personen und gruppendynamischen bzw. mikropolitischen Prozessen womöglich ein viel größerer Stellenwert zu, als dies geboten erscheint, und die Orientierung an der Sache gerät in den Hintergrund - allen heilsversprechenden agilen Diagnosen einer ausschließlich am Zweck ausgerichteten Organisation (» purpose-driven organization «; Fink und Moeller 2018) zum Trotz. Speziell in Krisensituationen können dadurch Unsicherheitszonen entstehen und Machtkämpfe auftreten (Stefan Kühl, zit. in Reimann 2017, 19; Kühl 2017, 23-26; zu mikropolitischen Taktiken von Ameln und Heintel 2016, 91-98 und Lang 2014, 189-194).

Das Diktum des Führungsforschers Abraham Zaleznik (2009, 87-89) - »It is precisely the job of leadership to create commitments that override the immediacy of personal interests [...] to keep politics out and substance in « - dürfte für formale wie informale Organisationen in gleicher Weise gelten. Doch wer sorgt für dessen Einhaltung? »Auch in agilen Organisationen braucht es Leadership - und damit zumindest temporäre Hierarchien « (Reimann 2017, 19), denn die Basisfunktionen, die Führung 
erfüllt, bleiben erhalten. Diese müssen sich an irgendwen emergent »anheften « bzw. irgendeine(r) muss sie aktiv aufgreifen und übernehmen. Dessen ungeachtet ist Führung in einem umfassenden Sinne immer als Kollektivphänomen zu begreifen. Die Lösung von Führungs- und Koordinationserfordernissen kann nicht allein auf den Stil oder das Rollenverhalten einer Führungsperson reduziert werden, sondern sie hat das Verhalten aller Gruppen- bzw. Organisationsmitglieder zu berücksichtigen, »die sich wechselseitig beeinflussen; es kommt auf alle an « (Scholl 2020, 146 - sinngemäß auch zum ersten Satzteil).

Tendenziell lässt sich sicherlich behaupten, dass informale Macht im Vergleich zur formalen Macht in agilen Organisationen eine größere Rolle spielt als in rein hierarchisch strukturierten Organisationen (von Ameln 2018, 28), wo das Verhältnis in der Regel umgekehrt ist. Durch das stärkere Gewicht der informalen Macht kommt es zu einer Neuausrichtung des klassischen Führungsverständnisses: Kontrolle wird zur Iteration, das heißt zu einer »sozialen Funktion « (Hensen 2020, 227-228), Struktur verändert sich zu Flexibilität und Transparenz, Misstrauen wird zu Vertrauen, Zentralisierung wird zu Dezentralisierung und Hierarchie wird zu Demokratie (Dörr et al. 2018, 40). Dennoch lassen sich weder in formalen Organisationen informale Machtphänomene noch in informalen Organisationen formale Machtphänomene je restlos zurückdrängen, weil jede Machtform Defizite aufweist, die nur die jeweils andere Machtform zu beseitigen imstande ist (vgl. auch Kühl 2017, 47). Die Frage lautet daher eher, wie sich das Zusammenspiel zwischen beiden Machtfeldern sinnvoll gestalten lässt. Im Hinblick auf Agilität stellen sich angesichts dieser stets vorhandenen und dynamisch entfaltenden Machtkonstellation folgende zwei Grundfragen:

$>$ Organisationale Ebene: Betrifft die Umsetzung von Agilität die gesamte Organisation oder lediglich bestimmte Teilbereiche (z. B. Entwicklungsteams, F\&E-Bereich)? Liegt eine echte agile Organisation mit umfassendem Empowerment der Mitarbeiter*innen und Teams vor oder eine unechte agile Organisation, in der die agilen Strukturen dem Einfluss der rahmengebenden hierarchischen Organisation mehr oder weniger stark ausgesetzt bleiben? Üben die ehemaligen Träger formaler Macht also weiterhin offen oder verdeckt Druck aus oder sind sie tatsächlich bereit, auf die Ausübung ihrer Macht zu verzichten?

$>$ Teamebene: Wie gestalten sich informale Macht- bzw. Einflussprozesse in agilen Teams im Zeitverlauf und welche theoretischen Ansätze helfen dabei, diese besser zu verstehen? Entwickeln sich speziell im Bereich der Führung am Ende doch wieder asymmetrische, quasi-hierarchische Einflussstrukturen, weil nicht jeder führen kann und will?

So wichtig die Auseinandersetzung mit beiden Fragen ist, werden wir die gesamtorganisationale Ebene hier nur kurz skizzieren. Unser Fokus liegt im Folgenden auf der 
Teamebene. Teams spielen in agilen Organisationskonzepten eine zentrale Rolle und sie sind auch jener Ort, an dem sich Macht- und Beeinflussungsprozesse vornehmlich abspielen.

\subsection{Agilität und Macht auf der organisationalen Ebene}

Allgemein geht es bei Agilität stets um eine schnellere Anpassungsfähigkeit der Organisation an die Umwelt, eine konsequente, iterativ-projektbegleitende Ausrichtung an den Kundenbedürfnissen (im Gegensatz zum früheren Wasserfallmodell), aber auch um eine echte Orientierung am Menschen, seinen Bedürfnissen und seinen Fähigkeiten. Dies gilt speziell für den Scrum-Ansatz und das Entscheidungsmodell der Soziokratie. Die Qualität der Beziehungen nach innen und nach außen wird zum entscheidenden Erfolgskriterium. Agilität ist damit in den größeren Trend der Demokratisierung, der kooperativen Führung, der zunehmenden Beteiligung von Mitarbeitern an unternehmerischen Entscheidungen und der Übertragung von (Führungs-)Kompetenzen auf nachgelagerte Stellen(-komplexe) (Empowerment) einzuordnen (Scholl 2020, 142-146 - zu einer Analyse älterer kooperativer bzw. emergenter Führungsmodelle). Sie steht in der Tradition der Human-Relations-Bewegung, der humanistischen Psychologie, der Organisationsentwicklung und folgt dem Trend des New Work. Eine eindeutige Definition für Agilität zu finden, erweist sich als schwierig. Es handelt sich eher um einen Sammelbegriff, der verschiedene ältere und neuere Prinzipien, Methoden und Maßnahmen der selbstorganisierten und flexiblen Arbeitssteuerung in Unternehmen bündelt.

Agilität versucht, ökonomische und humane Anliegen harmonisch zusammenzubringen. Konkret kann dies heißen: Verzicht auf unnötige Reportings; Vertrauensarbeitszeit; Mitarbeiter*innen wählen ihre Chefs selber; sie bestimmen ihr Gehalt im »kollegialen Consilium « und werden am Unternehmensgewinn beteiligt; Teams entscheiden eigenverantwortlich über ihr Handeln, ihr Budget und darüber, wer neu in das Team aufgenommen werden soll. Es kommt somit zu einer konsequenten Umsetzung des Subsidiaritätsprinzips - Entscheidungen werden dort getroffen, wo sie anfallen, Probleme dort gelöst, wo sie auftreten. Das Topmanagement hält sich aus dem operativen Geschehen heraus, lässt die selbstorganisiert arbeitenden Teams in Ruhe arbeiten und beschränkt sich auf die strategische Weiterentwicklung des Unternehmens. Unternehmen werden hierbei nicht selten zu einer Gemeinschaft, einer Ersatzfamilie und einem zweiten Zuhause, das oft weniger stressig ist als das eigentliche Zuhause (Hochschild 2006). Diese Gemeinschaft zeichnet sich durch Zusammengehörigkeitsgefühl, stärkenorientierte und flexible Zuweisung von Rollen, Commitment und ein hohes $\mathrm{Maß}$ an Hilfsbereitschaft und gegenseitiger Wertschätzung aus. 
Während bei »echten « agilen Unternehmen die ganzheitliche Umsetzung selbstorganisierter Agilität gelingt und ein über die Jahre gehender Kulturwandel (und damit Musterbruch) vollzogen wurde, zeigen sich in der Praxis viele Beispiele » unechter « agiler Unternehmen. Hier folgt das Management lediglich dem allgemeinen Modetrend, setzt Agilität in der Unternehmenspraxis aber nur halbherzig um. Es wird, ähnlich wie bei der Einführung von Lean-Management-Tools, auf die Entwicklung einer entsprechenden Kultur (Lean Thinking) verzichtet; ohne die im Alltag tatsächlich verankerte Mentalität und Praxis können die neuen Tools auf Dauer jedoch nicht erfolgreich sein (Hasenzagl und Link 2017) und alte Machtgefüge tauchen in neuem Gewand wieder auf.

\subsection{Agilität und Macht auf der Teamebene}

In der betrieblichen Praxis stellt sich die Machtfrage vor allem auf der Teamebene. Teams sind die entscheidenden Bauelemente zur Errichtung des » agilen Hauses «. Durch sie sollen individuelle Stärken aktiviert und Schwächen ausgeglichen, die Weisheit der Vielen genutzt und Synergien geschaffen werden. In selbstorganisiert arbeitenden Teams bzw. einem dynamischen Netzwerk solcher Teams in einem Unternehmen wird die Lösung all der in der hierarchisch-funktional gegliederten Struktur erkannten Unzulänglichkeiten gesehen (langwierige Entscheidungswege, fehlende Kooperationsorientierung, ein Mangel an interdisziplinärer Ausrichtung durch funktionale Abschottung, unzureichende Wissensbasis bei Entscheidungen). Entsprechend werden auf struktureller Ebene die Fähigkeit eines Unternehmens, sich gemäß den Umweltanforderungen in Teams immer wieder neu aufzustellen (»Teaming «), und auf personeller Ebene die Bereitschaft und das Potenzial zur Zusammenarbeit in Teams (»Teamfähigkeit «) zu entscheidenden Wettbewerbsfaktoren (Edmondson 2012, 2-3; Busch und von der Oelsnitz 2018, 41).

Gerade im Team Spirit - der engen Zusammenarbeit und dichten Kommunikation, der Nutzung individueller Stärken, der Aufbruchstimmung, dem besonderen Zusammenhalt und der sich dadurch dynamisch entfaltenden Kreativität - wird der Erfolg und die Innovationskraft junger und aufstrebender Unternehmen erkannt. Hinter all dem wollen Großunternehmen selbstverständlich nicht zurückstehen und ihr Bemühen um Agilisierung soll ebendiese belebenden Kräfte freisetzen.

Um das Einflussgeschehen in Teams zu verstehen, sollten zwei Axiome der Kommunikation von Paul Watzlawick in Erinnerung gerufen werden. Sein erstes, sicher berühmtestes Axiom ist: »Man kann nicht nicht kommunizieren.« Jegliche Form des kommunikativen Verhaltens (ob verbal oder non-verbal) hat demnach einen Mitteilungscharakter, zum Beispiel ist auch Schweigen eine durchaus nicht unbedeutende Form der Stellungnahme. Dieses Axiom lässt sich auch so verstehen, dass eine wechsel- 
seitige Einflussnahme in jedem Gespräch zwischen Menschen und damit auch in Teams zumindest wahrscheinlich ist. Als weniger bekanntes fünftes Axiom hat Watzlawick formuliert, dass zwischenmenschliche Kommunikationsabläufe entweder symmetrisch oder komplementär sind, je nachdem, ob die Beziehung zwischen den Partnern auf Gleichheit oder Unterschiedlichkeit beruht. In symmetrischen Beziehungen ist das Verhalten der Partner spiegelbildlich (z. B. in Bezug auf Stärke, Schwäche, Härte, Güte). Jeder verhält sich auf vergleichbare Weise. In komplementären Beziehungen ergänzt das Verhalten des einen Partners das des anderen. Ein Partner nimmt die superiore, primäre Stellung ein, der andere die inferiore, sekundäre (Mutter und Kind, Arzt und Patient, Lehrer und Schüler, Führungskraft und Geführter); das heißt jedoch nicht, dass die eine Seite stark und gut und die andere Seite schwach und schlecht ist (Watzlawick et al. 2011, 57-82). In komplementären Beziehungen entsteht etwa durch ein Wissensund Erfahrungsgefälle eine Informationsasymmetrie zwischen zwei Kommunikationspartnern, die dem superioren Teil mehr Einfluss einräumt und den inferioren Teil in die Position des Abhängigen versetzt.

In Teams kristallisieren sich solche komplementären Beziehungen in Gestalt einer Statusordnung mit der Zeit von ganz alleine aus. Statusverteilungen, die das eine Mitglied höher, das andere niedriger einstufen, finden permanent statt, wenn auch auf subtile Weise. Im Arbeitsalltag kommt es zu rangdynamischen Feinkalibrierungen, zu einem einflussbezogenen Kräftemessen zwischen einzelnen Teammitgliedern; zum Beispiel wird in einer Diskussion durch die Zuhörer und die Diskutanten sehr wohl registriert, wer letztlich »mehr Stiche « gemacht hat. Daraus resultiert dann mit der Zeit eine Statuseinschätzung und Rangzuweisung, die dem einen mehr, dem anderen weniger Einfluss im Kollektiv zubilligt. Diese Statuseinschätzungen unterscheiden sich jedoch aus Sicht einzelner Teammitglieder. Außerdem misst nicht jede(r) Statusfragen dieselbe Bedeutung bei. Sie unterliegen zudem Veränderungen. Auf- und Abstiege auf der imaginären Rangleiter sind im Zeitverlauf möglich, wobei auch die eigene Stellung von Team zu Team variieren kann. Anlässe für Rangverschiebungen liefern einzelne (un-)passende Bemerkungen, (un-)gerechte Verhaltensweisen und (Miss-)Erfolge der jeweiligen Bezugsperson. Diese kann ihren Status durch Role Making auch selbst aktiv zu vergrößern versuchen (etwa durch Impression Management, Signalling oder prosoziales, gruppenförderliches Verhalten).

Je nach wahrgenommenen Fähigkeiten wird Teammitgliedern Einfluss in unterschiedlichen Feldern in unterschiedlichem Ausmaß zugesprochen oder wieder aberkannt (z.B. im fachlichen, zwischenmenschlichen, führungsbezogenen, kreativen oder künstlerischen Bereich). Idealerweise deckt sich der wahrgenommene Status mit den zugewiesenen Aufgabenfeldern bzw. Rollen, die ein Teammitglied ausfüllt. Die Zuerkennung von Einfluss erfolgt aufgrund von drei zentralen Kräften (vgl. zu einer ähnlichen Einteilung Knoblach und Fink 2012, 754-759): 
$>$ individuelle Stärken (z. B. fundiertes Fachwissen, Talent),

$>$ Sympathie oder

$>$ Beziehungen, die das Mitglied im Team oder außerhalb des Teams hat.

Mit der Statuszuweisung aufgrund von Stärken beschäftigt sich die Rollentheorie, mit der Statuszuweisung aufgrund von Sympathien die Soziometrie und mit der Statuszuweisung aufgrund von Beziehungen die relationale Machttheorie. Mit diesen drei Ansätzen können Einflussphänomene besonders in agilen Organisationen greifbar gemacht und kritisch reflektiert werden.

\subsubsection{Rollentheorie: Einfluss über individuelle Stärken}

Speziell die funktionalistische Rollentheorie eignet sich, um das in agilen Strukturen zentrale Konzept der Rolle - spezifische Erwartungsbündel der Umwelt an den Rolleninhaber - zu verstehen. Gemäß diesem Ansatz müssen in jedem sozialen System (z.B. Kreis, Team) bestimmte Grundfunktionen erfüllt werden, damit das System arbeiten, seine Ziele erreichen und letztlich erhalten bleiben kann (Neuberger 1995, 982). Es ist hierbei unerheblich, von wem bestimmte Funktionen übernommen werden, wichtig ist nur, dass sie jemand erfüllt (Neuberger 2002, 327). Nach Belbin (2001) gibt es handlungs-, kommunikations- und wissensorientierte Rollen. Handlungsorientierte Rollen erfüllen Führungsfunktionen im Team, kommunikationsorientierte Rollen Sozialfunktionen und wissensorientierte Rollen inhaltlich-kreative Funktionen. Auch Driskell et al. $(2017,488)$ kommen in ihrer umfassenden Analyse zentraler Rollendimensionen in Teams zu einer ähnlichen Dreiteilung. Sie sprechen von Dominanzstreben, sozialem Streben und Aufgabenorientierung. Alle drei Grundfunktionen sollten in der Einflussstruktur eines Teams gleichmäßig abgebildet sein, damit dieses erfolgreich agieren kann. Ungleichgewichte (z. B. zu viel Dominanzstreben, zu viele kreative Meinungen) führen zu Leistungsverschlechterungen und Demotivation.

Die sich informal bildende machtbezogene Einflussstruktur wird durch das rangdynamische Rollenmodell von Raoul Schindler thematisiert. Schindler ging ebenfalls davon aus, dass erst ein strukturiertes und ausgeglichenes Ranggefüge ein Team nach innen stabil und nach außen erfolgreich macht. Dies setzt voraus, dass sämtliche Rollen kompetent besetzt sind. Für die Rollenbezeichnungen hat Schindler griechische Buchstaben verwendet. Alpha repräsentiert dabei die natürliche Führungsstärke, Beta die Stärke durch Wissen und Expertise, die Gammas stehen für das arbeitende Prinzip, das heißt für Handlungs- und Umsetzungsstärke, und Omega schließlich vertritt das »Gegengewicht « zu Alpha, nämlich Nonkonformität und Widerspruchsgeist. Er äußert Tabus und das, was sich Alpha nicht zu sagen traut. Durch die von ihm erzeugten Dissonanzen wird er häufig als Störenfried wahrgenommen, aber ohne ihn kann es 
zu ungesundem Harmoniestreben und kreativem Stillstand kommen (Schindler 2016, 232 sowie ausführlicher Busch und von der Oelsnitz 2018, 223-235).

Ein agiles Unternehmen ist entsprechend dieser Logik nur so gut wie die vorhandenen, sich ergänzenden Stärken seiner Mitglieder. Deren Nutzung und Kombination macht die unternehmerische Schlagkraft aus. Dies gilt allerdings auch für traditionell gegliederte Unternehmen, wie Peter Drucker schon vor über 50 Jahren überzeugend herausgearbeitet hat (Drucker 2014,79-98). Der Unterschied zu damals besteht darin, dass agile Organisationen von fixen Stellenzuweisungen abrücken und stattdessen flexible Rollenzuweisungen vornehmen. Der Grundgedanke hierbei ist, dass jeweils diejenige Person die temporäre Führung übernimmt, der in der spezifischen Situation die höchste Kompetenz zuerkannt wird. Dabei wird also auf ein modernes Führungsverständnis zurückgegriffen, das sich in der Literatur unter ganz unterschiedlichen begrifflichen Bezeichnungen findet, einerseits die Aufteilung der Führungsfunktionen, die »dynamische Delegation « (Klein et al. 2006) stärker hervorhebend (z.B. agile, laterale, emergente, plurale Führung, Shared Leadership, Distributed Leadership, Superleadership), andererseits die dienenden, entwicklungsorientierten und wertebasierten Funktionen der Führung, die als Facilitator bzw. Coach förderliche Strukturen herzustellen hat und unterstüzend wirkt (z. B. Servant, Positive, Transformational, Adaptive, Authentic, Ethical, Spiritual Leadership, systemische Führung).

Was genau Führung im Hinblick auf die oben genannten Machtbefugnisse konkret heißt und inwieweit sich der Führungsbedarf etwa im Laufe eines Projekts dynamisch ändert (Evans und Sanner 2019), bleibt in diesen Ansätzen zumeist recht vage. Im betrieblichen Alltag sollte Führung jedoch nicht vage gehalten werden, ansonsten droht Führungslosigkeit oder Führungsstreit. Inhaltliche Führung, die in den Ansätzen häufig hervorgehoben wird, ist beispielsweise etwas völlig anderes als disziplinarische Führung, die auch unliebsame Entscheidungen wie Abmahnung oder Entlassung mit einschließt. Das Vorbild der Führung allein genügt dann nicht. Entsprechend benötigen agile Organisationen Transparenz bei der Zuweisung inhaltlicher und disziplinarischer Befugnisse sowie klare Entscheidungs- und Kommunikationsregeln (inklusive der Möglichkeit von Sanktionen bei Nichteinhaltung) (Reimann 2017, 22). Auch informale, vermeintlich unbürokratische Organisationen haben so gesehen einen Formalisierungsbedarf und entwickeln konkrete Erwartungen. In der Scrum-Methode etwa haben sich klare Rollenprofile herausgebildet:

»Der Product Owner ist verantwortlich für die Beschaffenheit und Güte des Produkts [...]. Das Entwicklungsteam fertigt in der Umsetzung selbstbestimmt und eigenständig die inkrementellen Produkte. Der Scrum Master agiert im Sinne eines Servant Leaders als >Kümmerer $<$. Neben der Sorge für ein regel- und prinzipientreues Arbeiten [...] vermittelt er oder sie Methodenwissen, coacht im Interesse einer reibungslosen 
Zusammenarbeit und schafft angemessene Rahmenbedingungen « (Brückner und von Ameln 2016, 384).

Es ist damit zu rechnen, dass sich bei der Übernahme von Führungsfunktionen mit der Zeit eine Oligarchisierung - eine Herrschaft der Wenigen mit einem überproportionalen Einfluss - ergibt (Bluhm und Krause 2012 zu einer aktuellen Bestandsaufnahme des »ehernen Gesetzes der Oligarchie « nach Robert Michels), da in Menschen der »Wille zur Macht « bzw. das Machtmotiv, also die Bereitschaft zu führen - im Zentrum zu stehen, Verantwortung zu übernehmen und Einfluss auf andere auszuüben -, unterschiedlich stark ausgeprägt ist (McClelland und Burnham 1976; Pfeffer 2010), was zum Teil in familären Prägungen verwurzelt sein dürfte (Neubauer und Rosemann 2006, 62-66). Dies ist auch aus einer rein funktionalen Logik erklärbar, um ein mögliches Chaos » organisierter Verantwortungslosigkeit « (Rudolf Bahro) zu verhindern. Die auf dem Weg zur Oligarchie wirksamen Mechanismen, die am Ende dazu führen, dass » alle gleich, aber manche gleicher als die anderen sind «, hat George Orwell in seiner 1945 erschienenen dystopischen Fabel Animal Farm in literarischer Form zeitlos verarbeitet. Es ist kaum anzunehmen, dass ein agiles Unternehmen dieses Machtgesetz auf Dauer wird aushebeln können, speziell dann nicht, wenn es wächst und seinen $\gg$ Start-up-Charakter « verliert.

Dies ist der Grund, weshalb das Unternehmen W.L. Gore \& Associates ab einer bestimmten Größe »Zellteilung « betreibt, um die direkte und spontane Kommunikation zwischen Mitarbeiter*innen zu erhalten. Jeder soll jeden kennen. Wenn mehr als 150 bis 250 Menschen in einem Werk zusammenarbeiten, dann wird es in autonome Einheiten aufgeteilt. Ein gewisses Maß an Redundanz bei den Stellen wird dabei bewusst in Kauf genommen (Kaduk et al. 2020). Entscheidend ist demnach, dass trotz fast unvermeidbarer Oligarchisierungstendenzen (wobei die »Gleicheren « in agilen Organisationen von den anderen gewählt und respektiert werden) der Gemeinschaftsgeist in einem Unternehmen erhalten bleibt. Ansonsten drohen die aus Matrixorganisationen bekannten Dysfunktionen. Durch die Aufteilung von Führungsaufgaben auf unterschiedliche Rollenträger sind Abstimmungsprozesse und dabei entstehende Reibungen fast unabwendbar. Im guten Fall führt dies zu Synergien, Lernprozessen in Teams und einer hohen Problemlösungsfähigkeit, im schlechten Fall kommt es zu Intransparenz, Verzögerung von Entscheidungen (zu viel Gerede, zu wenig Ergebnisse), hohen Koordinationskosten (zu viele Manager, zu wenige Akteure), persönlicher Belastung (man wird zwischen den Fronten zerrieben) und übertrieben aufwendiger Dokumentation (Papierkrieg durch die vielen protokollierten Meetings) (Schreyögg und Geiger 2016, 95). Häufige Konflikte und Machtkämpfe durch die bewusst offengehaltenen Kompetenzabgrenzungen sowie die schwierige Zurechnung von Erfolgen und Misserfolgen können am Ende bei den Beteiligten zu Stress und Frustration mit erhöhtem Burn-out- 
Risiko führen. Auch agile Organisationen sind prinzipiell für derartige Negativerscheinungen anfällig, sofern das Wir-Gefühl abhandenkommt und keine klaren Regeln und allgemein akzeptierten Rollenzuweisungen existieren. Hinzu kommen die bekannten Schattenseiten der Teamarbeit (z. B. Mobbing, Groupthink, Leistungszurückhaltung, zu hoher Leistungs- oder Konformitätsdruck, erhöhte Risikoneigung) (Busch und von der Oelsnitz 2018, 211-213).

Ein weiteres, nicht wirklich gelöstes Problem ergibt sich aus der Rotation von Führungsrollen in agilen Teams. Darin tritt das gruppendynamische Spannungsfeld zwischen Nähe und Distanz in Erscheinung. Einmal nimmt eine Person die Rolle einer Vorgesetzten, das andere Mal die Rolle einer Kollegin oder Untergebenen ein. Speziell die Wahrnehmung eines Teammitglieds als Kolleg*in, das in einer unechten agilen Organisation auch eine formale Führungsposition innehat, aber auch dessen Selbstwahrnehmung, ist komplex und erzeugt Widersprüchlichkeiten. Unbewusst kann durch diese Rollenambiguität eine verdeckt hierarchische Verhaltens- und Meinungslenkung im Team erfolgen. Außerdem führt die temporäre Übernahme einer mit Machtbefugnissen ausgestatteten Rolle generell zu Änderungen im eigenen Erleben und Handeln, wobei dies weitgehend unbewusst vonstattengeht und von den Betroffenen unterschätzt wird (von Ameln 2018, 30). Eine offene Gesprächs- und Feedbackkultur, ein regelmäßiger Abgleich von Selbst- und Fremdbild und das lernförderliche Gefühl der psychologischen Sicherheit können diese Effekte verringern (Edmondson 1999).

Auch die bekannte, herausfordernde Konstellation des Übergangs von einer Kolleg*innen-Rolle in eine Lead-Rolle bleibt in agilen Organisationen bestehen. Wenn er oder sie sich nicht auf hierarchische Macht stützen kann, dann läuft die Möglichkeit zur Einflussnahme ausschließlich über die Erlangung von Autorität. Die Gefahr, sich hierbei durch eine übertriebene Form der Zuwendung und Unterstützung für das Team selbst auszubeuten, ist gegeben (Sichler 2006, 69-70). Bekanntlich nahm das Burn-outPhänomen in pflegenden Berufen seinen Ausgangspunkt. Die Entwicklung eines Helfersyndroms und eines Mangels an Abgrenzung ist bei einem rein dienenden Führungsverständnis, wie es in der agilen Philosophie vorgesehen ist, nicht unwahrscheinlich.

Schließlich kann sich auch so etwas wie ein »rangdynamischer Stress « entfalten. Wenn es keine klassischen Aufstiegschancen in der Hierarchie mehr gibt, sondern allenfalls noch die Möglichkeit der Gehaltssteigerung oder Prämienerlangung, dann kann ein eher psychologisch ausgetragener Wettbewerb um Status und Einfluss entbrennen. Es darf hierbei nicht übersehen werden, dass das Wettbewerbs- und Profitdenken tief in unserem westlichen Gesellschafts- und Bildungssystem verankert ist (vgl. hierzu den Film Alphabet - Angst oder Liebe? [2013] von Erwin Wagenhofer). »Everybody wants teamwork, but it's rarely successful because the deep cultural incentive system is still individualized « (Schein und von Ameln 2019, 143). Wenn sich die Entlohnungs- 
formen stärker am Team orientieren und der individuelle Anreiz über Gewinn- oder Umsatzbeteiligung erfolgt, so können die per se nicht schlechten kompetitiven Kräfte gemildert werden, ohne dass sie dabei gänzlich verloren gehen (Müller-Camen et al. 2019, 86-87).

All dies sind jedoch Probleme, die in agilen Strukturen durch den Kooperationsgedanken, durch Transparenz und durch Reflexionsprozesse erkannt und gelöst werden können, indem idealerweise nicht nur im Team, sondern auch kontinuierlich am Team (und an der Organisation) gearbeitet wird. Dazu zählt auch die Erörterung gesundheitlicher Fragen wie Burn-out-Prävention, Achtsamkeit und Resilienz. Die Teamforschung nennt die Fähigkeit zur kritischen Analyse von Teamprozessen und Teamstrukturen Teamreflexivität (West 2012, 10-11; Busch 2015, 106-146). »Die Fähigkeit, derartige Prozesse und sich selbst als Teil davon zu thematisieren, kann nicht als selbstverständlich vorausgesetzt werden. Sie bedarf eines eigenen Lernweges sowohl für das Individuum als auch für Teams und Organisationen « (Geramanis 2014, 187).

\subsubsection{Soziometrie: Einfluss über Sympathie}

Neben den wahrgenommenen individuellen Stärken und der Arbeitsleistung spielt bei der Frage, inwiefern ein Mensch in der Lage ist, Personen in seiner Umwelt wirksam zu beeinflussen und zu überzeugen, der Aspekt eine Rolle, als wie sympathisch er wahrgenommen wird. Je (un-)sympathischer uns ein Mensch erscheint, desto eher (weniger) sind wir bereit, Feedback (Ratschläge oder Kritik) von ihm anzunehmen, und zwar unabhängig davon, ob dieses Feedback berechtigt ist oder nicht. Deswegen wird in agilen Organisationen auch so viel Wert auf das passende Mindset - Persönlichkeit, Integrität und Haltung - bei Rekrutierungsentscheidungen gelegt (im Sinne des »hire character, train skills «). Oft entscheiden die Teams, in denen der oder die Neue arbeiten soll, nach einer Probezeit selbst über die Aufnahme (Busch und Lorenz 2009). Die »Chemie « - die zwischenmenschliche Passung - soll stimmen, denn wechselseitige Sympathie führt zu qualitativ hochwertigen Beziehungen, zu Kreativität, Vertrauen, Leistungsbereitschaft, ja Glücksempfinden am Arbeitsplatz (Moreno 1996, 438-440; Busch 2019, 52). Kriterien wie Intelligenz, Fachwissen und Motivation dürfen darüber natürlich nicht vergessen werden.

Die Messung von Sympathie in bestehenden Arbeitsbeziehungen erfolgt über Wahl oder Nichtwahl, lässt sich anonym erfragen und grafisch in Soziogrammen abbilden. Dabei kann unter anderem eine Tüchtigkeitsrangordnung (Mit wem würden Sie am liebsten zusammenarbeiten?) oder eine Beliebtheitsrangordnung (Neben wem würden Sie am liebsten bei einem Betriebsausflug sitzen?) erfasst werden. Auf Sympathie beruhende Einflussphänomene lassen sich auf diese Weise gut abbilden. Dies gilt auch für die Wahrnehmung der Führungsfähigkeit. Die Führungsrolle erscheint hierbei als 
sympathiebasiertes Phänomen: Zur Führungsperson wird jemand dann, wenn er oder sie durch die Wahlen anderer dazu gemacht wird (von Ameln 2014, 213-215). Ein populärer Führer erhält dabei viele Wahlen von Teammitgliedern mit niedrigem soziometrischem Status, ein mächtiger Führer viele Wahlen von Personen mit hohem soziometrischem Status (Moreno 1996, 172-173). Rein ökonomisch sagt die Beliebtheit aber noch nichts über den Führungserfolg aus (Busch und von der Oelsnitz 2018, 222). Bereits in interaktionsanalytischen Studien aus den 1950er Jahren konnte zudem festgestellt werden, dass eine funktionierende Gruppenordnung stets beide Kräfte in sich vereinen bzw. beide Rollen besetzt haben sollte - die des (eher unbeliebten) $\gg$ Task Leader « und die des (eher beliebten) »Social-emotional Leader « (Bales und Slater 1955). Selten vereint eine Person beide Gestaltungspotenziale in sich; fehlt ihr zum Beispiel die Fähigkeit zur emotionalen Aktivierung, so sollte zumindest ein Teammitglied in der Lage sein, dies auszugleichen, um den Zusammenhalt in der Gruppe aufrechtzuerhalten.

\subsubsection{Relationale Machttheorie: Einfluss über Beziehungen}

Sympathien spielen auch eine wichtige Rolle bei der Formierung von Beziehungen. Beziehungen sind nicht nur durch die positive Psychologie als elementar für das Wohlergehen eines Menschen erkannt worden, sondern sie bilden zugleich eine wichtige Machtgrundlage, denn über gute Beziehungen im Team und ein Netzwerk an Kontakten außerhalb des Teams kann ein Teammitglied Wissen und Unterstützung mobilisieren. Durch dieses »Sozialkapital « (Bourdieu 1983, 191-193) können dem Team wertvolle Informationen zufließen oder Hilfestellungen zuteilwerden (Busch und von der Oelsnitz 2018, 61). Teammitglieder, die über entsprechende Kontakte nach innen und außen verfügen, erlangen auf diese Weise einen größeren Einfluss, wobei Macht hier eher in einem relationalen Sinne existiert (Kühl 1998, 94-95). Sie lässt sich also nicht eindeutig lokalisieren und steckt im Kräfteverhältnis von Beziehungen (König 1998, 19; Foucault 2005, 240; Sichler 2006, 217-230), das heißt, sie ist » untrennbar mit den Personen und der sozialen Praxis verwoben « (von Ameln und Heintel 2016, 31-32). Macht wird also »in der Interaktion der Beteiligten gemeinsam konstruiert « (von Ameln 2018, 32). Wer ein stärkeres Netzwerk an Beziehungen in einem Team oder einer Organisation hat, kann Koalitionen bilden und Mehrheitsverhältnisse beeinflussen. Er oder sie verfügt zudem über eine höhere Definitions- und Deutungsmacht, indem er oder sie darüber bestimmen kann, » wie eine Situation > richtig< zu interpretieren ist und was die daraus zu ziehenden $>$ korrekten $<$ Schlussfolgerungen sind « (Busch und von der Oelsnitz 2018, 222). Allein das Wissen, dass hinter einer Person mächtige andere Personen stehen, beeinflusst deren Wahrnehmung durch die Mitmenschen und löst bei diesen ein verändertes, mitunter unterwürfiges Verhalten aus. 
Für die Erklärung von Beziehungsmustern innerhalb von Organisationen ist das Konzept des transaktiven Gedächtnisses bedeutsam. Damit ist das sich im Zeitverlauf natürlich bildende oder durch Entwicklungsmaßnahmen bewusst herbeigeführte (Meta-)Wissen über das Wissen von Teamkolleg*innen und Organisationsmitgliedern gemeint (Wegner 1987; Busch 2008, 41-49). Zu wissen, wer was weiß und kann, wie der- oder diejenige $\gg$ tickt «, ist nicht nur eine wesentliche Grundlage für die wechselseitige Verständigung und die Verbesserung von Informations-, Koordinations- und Problemlösungsprozessen (Schließung eigener Wissenslücken), sondern bildet auch die Basis für die Aktivierung von Koalitionen in Entscheidungsprozessen. Je länger jemand in einer Organisation arbeitet, desto feinkörniger ist sein bzw. ihr Personen- und Beziehungswissen. Zu diesem Wissen zählt auch der zuvor erörterte Sympathieaspekt. Es ist nicht nur hilfreich (und einflussfördernd) zu wissen, wer welche Fähigkeiten hat, sondern auch zu wissen, wer mit wem »kann « und wer sich mit wem verbinden lässt, um bestimmte Ziele durchzusetzen. Oft bildet ein solches Wissen (speziell über die äußeren Kontakte und Netzwerke) eine Art »Geheimwissen «. Agile Organisationen sind dagegen darum bemüht, auch in diesem Bereich größtmögliche Transparenz herzustellen, um nicht Seilschaften oder »Old Boys' Networks « entstehen zu lassen, sondern partnerschaftliche Verbindungen, die allen zugutekommen.

In hierarchischen Organisationen bilden systematische Offenlegungen dieses Machtwissens eher die Ausnahme, da die wenigsten dazu bereit oder überhaupt in der Lage sind, völlige Transparenz über ihre Innen- und Außenkontakte herzustellen. Netzwerkwissen erschließt sich eher zufällig durch die Zusammenarbeit, zum Beispiel bei auftretenden Problemen, für die das Team keine Lösung findet. Andererseits bieten soziale Netzwerke heutzutage sehr viel leichter als früher die Möglichkeit, Einblick in die Freundeslisten von Kolleg*innen zu bekommen (Busch und von der Oelsnitz 2018, 62). Mit der Erfassung und Bewertung dieses Beziehungswissens beschäftigt sich in der Wissenschaft die Netzwerktheorie. In der Netzwerkanalyse wird die Einbindung eines Akteurs in organisationsinterne und -übergreifende Netzwerke systematisch erfasst und visualisiert (Wassermann und Faust 1994). Zentrale Messgrößen, die Aufschluss über die relationale Einflussmacht eines Akteurs geben, sind hierbei (Katz et al. 2005, 280-281):

$>$ Der Zentralitätsgrad eines Akteurs: Wie viele Verbindungen gehen von einem Akteur aus bzw. laufen bei ihm zusammen?

$>$ Die Zwischenzentralität: Über wen laufen die meisten Informationen?

$>$ Die Nähezentralität: Wie stark sind Akteure direkt und indirekt mit dem Rest des Netzwerks verbunden?

$>$ Die Reziprozität: Inwiefern beruhen Beziehungen auf Gegenseitigkeit?

$>$ Die Transitivität: Inwiefern sind Akteure, die miteinander verbunden sind, in gleicher Weise mit anderen verbunden? Im Facebook-Jargon: Ist der Freund eines Freundes auch mein Freund? 
Kritisch zu fragen ist, wie realistisch die Umsetzung einer solchen Offenlegung im Alltag ist und ob die Bereitschaft hierzu bei den Mitarbeiter*innen tatsächlich vorhanden ist (von rechtlichen Bedenken bezüglich Datenschutz einmal abgesehen). Ob gewollt oder nicht: Auf Dauer werden sich auch in agilen Organisationen Einflusscluster und freundschaftliche Verbindungen aufgrund langjähriger Zusammenarbeit etablieren. Sympathiegefühle lassen sich schließlich nicht einfach » wegorganisieren «. Speziell für Neuzugänge in Organisationen ist es schwierig, in solche Ingroups bzw. »Netzwerke der Eingeweihten « (Schrader und Wenzl 2015, 138) mit eigenen Sprachcodes und gemeinsamen Erlebnissen aus der Vergangenheit vorzudringen. Das ist eine Herausforderung für das Onboarding. Dieses sollte neue Mitarbeiter*innen nicht nur über die Arbeit integrieren, sondern auch eingehend mit der Kultur und den betrieblichen Gemeinschaften vertraut machen.

Beziehungen haben jedoch auch noch eine weitere, subtiler wirkende Tiefendimension, die der französische Soziologe Pierre Bourdieu im Konzept des Habitus gründlich erfasst hat (Bourdieu 1982; von Ameln und Heintel 2016,28-30). Dieser kann ebenfalls zu einem möglichen Ausschlusskriterium für die inoffizielle Zugehörigkeit in bestimmten Gruppen werden. Der Habitus, das heißt die durch die Schichtzugehörigkeit, die Art der Erziehung und (Aus-)Bildung anerzogene, in der Sprache, Körperhaltung, Kleidung und im Geschmack zum Ausdruck kommende Wesensart führt zu unsichtbaren Rangunterschieden zwischen Individuen. Er schafft $»$ feine Unterschiede «, die im Alltag gleichwohl grobe $\mathrm{Ab}$ - und Ausgrenzungen nach sich ziehen können. Der Habitus gibt - in den Worten Bourdieus - dem Inhaber neben dem ökonomischen besonders durch das inkorporierte kulturelle und soziale Kapital Jetons in die Hand, die er im »Casino des Lebens « auf den Spieltisch legen kann (oder eben nicht). Echte Gleichheit (im Gegensatz zur Gleichberechtigung) wird es daher nie geben können. Durch habituelle Ähnlichkeiten entstehen in jedem sozialen System Koalitionen und Netzwerke - filigran wirkende Machtzonen. Macht im Sinne von Einflussmöglichkeiten und Einflussgewährung bahnt sich auf diese Art im Verborgenen ihren Weg.

Zusammenfassend gibt es in der agilen Organisation über Differenzen in der individuellen Stärke, über die gemeinschaftsbildende (oder -verhindernde) Wirkung von Sympathie und über den Aufbau von Beziehungen genügend Kräfte, die am Ende doch wieder zur Herausbildung einer sich eher verschleiert, hintergründig aufsetzenden Rangordnung führen (vgl. Tabelle 3). In dieser jedoch nicht unabänderlichen Ordnung entscheidet die jeweilige Statusposition darüber, wie viel Einfluss der Ranginhaber in der Organisation ausüben kann und darf. Diese Ordnung gleicht in den Worten der Systemtheorie einem funktionalen Äquivalent, das die vormals hierarchischen Mechanismen der Machtdurchsetzung ersetzt. Nach systemtheoretischem Verständnis erfüllt die Hierarchie ganz bestimmte Grundfunktionen (z.B. zielgerichtete Lenkung von Organisationsmitgliedern, Entscheidungsbildung, Konfliktlösung). Wird Hierarchie 


\begin{tabular}{|c|c|c|c|}
\hline \multicolumn{4}{|c|}{ Informaler Einfluss in agilen Organisationen } \\
\hline Einflussgrundlage & $\begin{array}{l}\text { Persönliche Stär- } \\
\text { ken (Arbeitsleis- } \\
\text { tung, Talent) } \\
\text { KÖNNEN }\end{array}$ & $\begin{array}{c}\text { Sympathie } \\
\text { (Zu- oder Abnei- } \\
\text { gung) } \\
\text { MITEINANDER } \\
\text { KÖNNEN }\end{array}$ & $\begin{array}{l}\text { Beziehungen } \\
\text { (im Team und dar- } \\
\text { über hinaus) } \\
\text { JEMANDEN } \\
\text { KENNEN }\end{array}$ \\
\hline $\begin{array}{l}\text { Theoretischer Er- } \\
\text { klärungsansatz }\end{array}$ & $\begin{array}{l}\text { Funktionalistische } \\
\text { Rollentheorie } \\
\text { Gleichmäßige Er- } \\
\text { füllung arbeits- } \\
\text { und kooperations- } \\
\text { relevanter Grund- } \\
\text { funktionen (Hand- } \\
\text { lungs-, Kommuni- } \\
\text { kations- und Wis- } \\
\text { sensorientierung) }\end{array}$ & $\begin{array}{l}\text { Soziometrie } \\
\text { Sympathiebasierte } \\
\text { Teambildung (Er- } \\
\text { fassung über Wahl- } \\
\text { oder Nicht-Wahl- } \\
\text { Entscheidungen in } \\
\text { Soziogrammen) }\end{array}$ & $\begin{array}{c}\text { Relationale } \\
\text { Machttheorie } \\
\text { Macht als sozia- } \\
\text { les Phänomen, als } \\
\text { aktivierbares Netz- } \\
\text { werk an Kontakten } \\
\text { (Sozialkapital) }\end{array}$ \\
\hline Einflussausmaß & $\begin{array}{l}\text { Abhängig von der } \\
\text { erfolgreichen Be- } \\
\text { wältigung übertra- } \\
\text { gener Rollen }\end{array}$ & $\begin{array}{l}\text { Abhängig von der } \\
\text { Fähigkeit, Gefühle } \\
\text { der Zuneigung in } \\
\text { der Umwelt her- } \\
\text { vorzurufen }\end{array}$ & $\begin{array}{l}\text { Abhängig von der } \\
\text { Möglichkeit, } \\
\text { mächtige Koalitio- } \\
\text { nen zu schmieden } \\
\text { und Mehrheiten in } \\
\text { Entscheidungen } \\
\text { zu lenken }\end{array}$ \\
\hline
\end{tabular}

Tab. 3: Informaler Einfluss in agilen Organisationen

abgebaut, entsteht zwangsläufig eine Leerstelle. Der funktionale Bedarf bleibt erhalten und muss auf andere Weise gedeckt werden. Die agile Einflussordnung kann dies leisten, aber auch sie muss sich entsprechend formieren und durch selbstorganisierte Lern-, Koordinations-, Kontroll- und Reflexionsprozesse immer wieder reformieren. Dies gelingt auf Dauer nur, wenn der agile Geist lebendig beibt und sich natürliche oligarchische Tendenzen nicht in neuen (Quasi-)Hierarchien verfestigen, in denen Kritik, offener Dialog und der Wettbewerb um die bessere Idee allmählich verloren gehen.

\section{$4 \quad$ Fazit}

Das Spannungsverhältnis zwischen formaler und informaler Macht ist ein seit den Hawthorne-Studien bekannter Forschungsgegenstand. Während in der klassisch-hierarchisch strukturierten Organisation der formalen Macht größeres Gewicht als der informalen Macht zukommt, wird in agilen Organisationen primär auf die Kraft der Überzeugung durch informale Macht und demokratische Willensbildung gesetzt. Doch 
auch hier sind Regeln und hierarchieähnliche »Letztentscheidungen« erforderlich. Wie genau sich dies im Einzelfall auszugestalten hat, ist durch die jeweiligen kontextuellen Gegebenheiten und Restriktionen zu klären. Gewiss ist nur, dass es Agilisierung » nicht von der Stange [gibt]. Die richtige Organisationsform muss gesucht, erprobt und angepasst - und notfalls auch wieder abgeschafft - werden « (Bußmann, 2017, $3)$. Von der Vorstellung eines » one best way« oder einer idealen Organisation sollte unbedingt Abstand genommen werden. Es gibt nur mehr oder weniger passende Strukturen, die durch Umweltveränderungen wieder zu ungeeigneten Strukturen werden können. Gerade einseitige Ausgestaltungen der Organisation erweisen sich häufig als problematisch (Grundei und Kaehler 2018, 431). Diese kontingenztheoretische Ureinsicht sollte bei aller Euphorie für neuartige Ansätze stets im Hinterkopf bewahrt werden. Dabei darf speziell der Faktor Macht - ob in formaler oder informaler Gestalt auftretend - nie unterschätzt werden. Er kann utopische Vorstellungen organisationaler Demokratien durchkreuzen, nur zu oligarchischen Scheindemokratien führen oder partizipative Modelle gänzlich zunichtemachen. Wie gezeigt, handelt es sich bei Macht um ein allgegenwärtiges, » unkaputtbares « Phänomen des zwischenmenschlichen Miteinanders; entsprechend tritt sie auch immer in Erscheinung - unabhängig vom gewählten organisationalen Gestaltungskonzept. Agile Organisationen bilden hier keine Ausnahme. Umso wichtiger ist es daher, die Wirkungsmechanismen von Macht zu verstehen, sie transparent zu machen und unterschiedliche Einflussformen je nach situativen Erfordernissen zu bewerten und sinnvoll einzusetzen. Manchmal ist es in agilen Organisationen notwendig, im Sinne der demokratischen Entscheidungsfindung den eigenen Willen zur Macht zu zügeln. Agilität funktioniert so lange, wie der Gemeinschaftssinn, der Teamgedanke, das füreinander Einstehen, die Freude an der Verfolgung gemeinsamer Ziele und die freiwillige Zuweisung von Machtrollen gegeben sind und fortlaufend kultiviert werden.

\section{Literatur}

Ameln, Falko von. 2014. »Mensch und Organisation. Morenos Werk aus der Sicht der Organisations- und Führungsforschung «. Zeitschrift für Psychodrama und Soziometrie 13 (1): 199-223.

Ameln, Falko von. 2018. »Agilität und Führung - eine Frage der Macht«. Wirtschaftspsychologie aktuell 25 (2): 28-34.

Ameln, Falko von und Peter Heintel. 2016. Macht in Organisationen. Denkwerkzeuge für Führung, Beratung und Change Management. Stuttgart: Schäffer-Poeschel.

Ameln, Falko von und Josef Kramer. 2012. »Macht und Führung. Gedanken zur Führung in einer komplexer werdenden Organisationslandschaft«. Gruppendynamik und Organisationsberatung 43 (2): 189-204.

Bales, Robert F. und Philipp E. Slater. 1955. »Role Differentiation in Small Decision-Making Groups«. In Family, Socialization, and Interaction Processes, hrsg. v. Talcott Parsons und Robert F. Bales, 259-306. Glencoe: The Free Press. 
Baumann-Habersack, Frank. 2017. Mit neuer Autorität in Führung. Die Führungshaltung für das 21. Jahrhundert. 2. Aufl. Wiesbaden: Springer Gabler.

Belbin, R. Meredith. 2001. Team Roles at Work. Oxford: Butterworth-Heinemann.

Bluhm, Harald und Skadi Krause, Hrsg. 2012. Robert Michels' Soziologie des Parteiwesens. Oligarchien und Eliten - die Kehrseiten moderner Demokratie. Wiesbaden: Springer VS.

Bourdieu, Pierre. 1982. Die feinen Unterschiede. Kritik der gesellschaftlichen Urteilskraft. Frankfurt a. M.: Suhrkamp.

Bourdieu, Pierre. 1983. »Ökonomisches Kapital, kulturelles Kapital, soziales Kapital«. In Soziale Ungleichheiten, hrsg. v. Reinhard Kreckel, 183-98. Göttingen: Schwartz.

Brückner, Fabian und Falko von Ameln. 2016. »Agilität«. Gruppe. Interaktion. Organisation. Zeitschrift für angewandte Organisationspsychologie (GIO) 47 (4): 383-86.

Busch, Michael W. 2008. Kompetenzsteuerung in Arbeits- und Innovationsteams. Wiesbaden: Gabler.

Busch, Michael W. 2014. »Die uniforme Organisation: Auszehrung der Mitarbeiter als Folge von Konformitätsdruck in Teams". In Die auszehrende Organisation. Leistung und Gesundheit in einer anspruchsvollen Arbeitswelt, hrsg. v. Dietrich von der Oelsnitz, Frank Schirmer und Kerstin Wüstner, 133-53. Wiesbaden: Springer Gabler.

Busch, Michael W. 2015. Management und Dynamik teambezogener Lernprozesse. München: Rainer Hampp.

Busch, Michael W. 2019. »Wer kann mit wem? Die Rolle von Sympathie in Teams«. Austrian Management Review 9: 46-56.

Busch, Michael W. und Marcus Lorenz. 2009. »Zusammenstellung von Projektteams - Mitarbeiter beteiligen und Wissen erfolgreich nutzen«. Personalführung 42 (7): 54-59.

Busch, Michael W. und Dietrich von der Oelsnitz. 2018. Teammanagement. Grundlagen erfolgreichen Zuammenarbeitens. Stuttgart: Kohlhammer.

Bußmann, Nicole. 2017. »Ist Hierarchie out?«. managerSeminare 236 (11): 3.

Dörr, Stefan, Pina Albo und Beate Monastridis. 2018. »Digital Leadership - Erfolgreich führen in der digitalen Welt«. In Führungsinstrumente aus dem Silicon Valley. Konzepte und Kompetenzen, hrsg. v. Rüdiger Goyk und Sven Grote, 38-58. Berlin: Springer Verlag.

Driskell, Tripp, James E. Driskell, Shawn Burke und Eduardo Salas. 2017. »Team Roles: A Review and Integration«. Small Group Research 48 (4): 482-511.

Drucker, Peter F. 2014. The Effective Executive. Effektivität und Handlungsfähigkeit in der Führungsrolle gewinnen. München: Vahlen.

Edmondson, Amy. 1999. »Psychological Safety and Learning Behavior in Work Teams«. Administrative Science Quarterly 44 (2): 350-83.

Edmondson, Amy C. 2012. Teaming. How Organizations Learn, Innovate, and Compete in the Knowledge Economy. San Francisco: Jossey-Bass.

Etzioni, Amitai. 1961. A Comparative Analysis of Complex Organizations. On Power, Involvement, and their Correlates. New York: The Free Press of Glencoe.

Evans, Karoline und Bret Sanner. 2019. „When Hierarchy Falls Flat: Temporal Changes in Hierarchy and when they Harm Performance«. Academy of Management Annual Meeting Proceedings 1: 13199.

Fink, Franziska und Michael Moeller. 2018. Purpose Driven Organizations: Sinn - Selbstorganisation Agilität. Stuttgart: Schäffer-Poeschel.

Fischer, Lorenz und Günter Wiswede. 2009. Grundlagen der Sozialpsychologie. 3. Aufl. München: OIdenbourg.

Foucault, Michel. 2005. Analytik der Macht. Frankfurt a. M.: Suhrkamp.

French, John R.P. und Bertram Raven. 1959. »The Bases of Social Power«. In Studies in Social Power, hrsg. v. Dorwin Cartwright, 150-67. Ann Arbor: The University of Michigan. 
Friedberg, Erhard. 1992. »Zur Politologie von Organisationen«. In Mikropolitik. Rationalität, Macht und Spiele in Organisationen, 2. Aufl., hrsg. v. Willi Küpper und Günther Ortmann, 39-52. Opladen: Westdeutscher Verlag.

Galbraith, John Kenneth. 1983. The Anatomy of Power. Boston: Houghton Mifflin.

Geramanis, Olaf. 2014. „Die Zukunft der Organisation. Kann man Teams vertrauen?«. In Organisation und Intimität. Der Umgang mit Nähe im organisationalen Alltag - zwischen Vertrauensbildung und Manipulation, hrsg. v. Olaf Geramanis und Kristina Hermann, 173-93. Heidelberg: CarlAuer.

Glogler, Boris. 2016. Scrum. Produkte zuverlässig und schnell entwickeln. 5. Aufl. München: Hanser.

Grundei, Jens und Boris Kaehler. 2018. »Wie erreichen Unternehmen mehr Agilität? Ein kritischer Blick auf ıneue` Formen der Organisation«. Zeitschrift Führung + Organisation 87 (6): 427-34.

Han, Byung-Chul. 2005. Was ist Macht? Stuttgart: Reclam.

Hasenzagl, Rupert und Karin Link. 2017. »Agil: Das neue Paradigma in der Unternehmensführung? Managementkonzepte für agile Organisationen«. Austrian Management Review 7: 47-55.

Hensen, Peter. 2020. »Die Rolle der Kontrolle in Organisationen: Konzeptionen und Nutzenapekte eines facettenreichen Begriffs«. Gruppe. Interaktion. Organisation. Zeitschrift für Angewandte Organisationspsychologie (GIO) 51 (2): 223-34.

Hobbes, Thomas. 1996. Leviathan. Hamburg: Felix Meiner.

Hochschild, Arlie Russell. 2006. Keine Zeit. Wenn die Firma zum Zuhause wird und zu Hause nur Arbeit wartet. 2. Aufl. Wiesbaden: VS.

Kaduk, Stefan, Dirk Osmetz, Hans A. Wüthrich und Dominik Hammer. 2020. Musterbrecher. Die Kunst, das Spiel zu drehen. Hamburg: Murmann.

Katz, Nancy, David Lazer, Holly Arrow und Noshir Contractor. 2005. »The Network Perspective of Small Groups. Theory and Research «. In Theories of Small Groups. Interdisciplinary Perspectives, hrsg. v. Marshall Scott Poole und Andrea B. Hollingshead, 277-312. Thousand Oaks: Sage.

Keltner, Dacher. 2016. »Macht ist ein Geschenk«. Psychologie heute 43 (11): 70-74.

Kieser, Alfred und Herbert Kubicek. 1992. Organisation. 3. Aufl. Berlin: de Gruyter.

Klein, Katherine J., Jonathan C. Ziegert, Andrew P. Knight und Yan Xiao. 2006. »Dynamic Delegation: Shared, Hierarchical, and Deindividualized Leadership in Extreme Action Teams«. Administrative Science Quarterly 51 (4): 590-621.

Knoblach, Bianka und Dietmar Fink. 2012. »Warum wir tun, was andere wollen: Psychologische Determinanten informeller Macht in Organisationen«. Schmalenbachs Zeitschrift für betriebswirtschaftliche Forschung 64 (7): 747-71.

König, Oliver. 1998. Macht in Gruppen. Gruppendynamische Prozesse und Interventionen. 2. Aufl. München: Pfeiffer.

Krogh, Georg von und Johan Roos. 1995. »A Perspective on Knowledge, Competence and Strategy«. Personnel Review 24 (3): 56-76.

Kühl, Stefan. 1998. Wenn die Affen den Zoo regieren. Die Tücken der flachen Hierarchie. 5. Aufl. Frankfurt a. M.: Campus.

Kühl, Stefan. 2017. Laterales Führen. Eine kurze organisationstheoretisch informierte Handreichung. Wiesbaden: Springer VS.

Landwehrmann, Friedrich. 1969. »Autorität«. In Handwörterbuch der Organisation, hrsg. v. Erwin Grochla, 269-73. Stuttgart: Carl Ernst Poeschel.

Lang, Reinhart. 2014. »Mikropolitischer Führungsansatz: Wer führt wen?«. In Aktuelle Führungstheorien und Konzepte, hrsg. v. Reinhart Lang und Irma Rybnikova, 181-212. Wiesbaden: Springer Gabler.

Luhmann, Niklas. 1999. Funktionen und Folgen formaler Organisation. 5. Aufl. Berlin: Duncker \& Humblot. 
Malik, Fredmund. 2006. Führen, Leisten. Leben. Wirksames Management für eine neue Zeit. Frankfurt a. M.: Campus.

McClelland, David C. und David H. Burnham. 1976. »Power is the Great Motivator«. Harvard Business Review 54 (2): 100-10.

Miebach, Bernhard. 2012. Organisationstheorie. Problemstellung - Modelle - Entwicklung. 2. Aufl. Wiesbaden: Springer VS.

Mitchell, Terence R. 1995. »Führungstheorien - Attributionstheorie«. In Handwörterbuch der Führung, 2. Aufl., hrsg. v. Alfred Kieser, Gerhard Reber und Rolf Wunderer, 847-61. Stuttgart: Schäffer-Poeschel.

Moreno, Jacob L. 1996. Die Grundlagen der Soziometrie. Wege zur Neuordnung der Gesellschaft. Wiesbaden: Springer.

Morgan, Gareth. 2008. Bilder der Organisation. 4. Aufl. Stuttgart: Schäffer-Poeschel.

Müller-Camen, Michael, Jürgen Weibler, Brian Matthews und Christoph Riess. 2019. »Transformationen im Personalmanagement: Die Beispiele Sonnentor und Tele Haase«. In Chancen und Grenzen der Nachhaltigkeitstransformation. Ökonomische und soziologische Perspektiven, hrsg. v. Fred Luks, 79-93. Wiesbaden: Springer Gabler.

Neubauer, Walter und Bernhard Rosemann. 2006. Führung, Macht und Vertrauen in Organisationen. Stuttgart: Kohlhammer.

Neuberger, Oswald. 1995. »Führungstheorien - Rollentheorie«. In Handwörterbuch der Führung, 2. Aufl., hrsg. v. Alfred Kieser, Gerhard Reber und Rolf Wunderer, 979-93. Stuttgart: SchäfferPoeschel.

Neuberger, Oswald. 2002. Führen und führen lassen. Ansätze, Ergebnisse und Kritik der Führungsforschung. 6. Aufl. Stuttgart: Lucius \& Lucius.

Nye, Joseph S. 1990. »Soft Power«. Foreign Policy 80 (3): 153-71.

Patchen, Martin. 1974. »The Locus and Basis of Influence on Organizational Decisions«. Organizational Behavior and Human Performance 11 (2): 195-221.

Pfeffer, Jeffrey. 1992. Managing with Power. Politics and Influence in Organizations. Boston: Harvard Business School Press.

Pfeffer, Jeffrey. 2010. Power. Why Some People Have it - and Others don't. New York: HarperCollins.

Popitz, Heinrich. 1992. Phänomene der Macht. 2. Aufl. Tübingen: Mohr.

Porschen-Hueck, Stephanie, Marc Jungtäubl und Margit Weihrich. 2020. Agilität? Herausforderungen neuer Konzepte der Selbstorganisation. Augsburg: Rainer Hampp.

Probst, Gilbert J. B. 1992. „Selbstorganisation«. In Handwörterbuch der Organisation, 3. Aufl., hrsg. v. Erich Frese, 2255-69. Stuttgart: Carl Ernst Poeschel.

Reimann, Sascha. 2017. »Agilisierung der Unternehmen. Das Ende der Hierarchie?«. managerSeminare 236 (11): 18-25.

Rifkin, Jeremy. 2000. Access. Das Verschwinden des Eigentums. Warum wir weniger besitzen und mehr ausgeben werden. Frankfurt a. M.: Campus.

Robertson, Brian J. 2016. Holacracy: Ein revolutionäres Management-System für eine volatile Welt. München: Vahlen.

Russell, Bertrand. 2000. Macht. Hamburg: Europa-Verlag.

Sandner, Karl. 1993. Prozesse der Macht. Zur Entstehung, Stabilisierung und Veränderung der Macht von Akteuren in Unternehmen. 2. Aufl. Berlin: Springer.

Schein, Edgar H. und Falko von Ameln. 2019. »Relationships as a Key to Change. Ed Schein on the Legacy and Future of Change Management«. Gruppe. Interaktion. Organisation. Zeitschrift für Angewandte Organisationspsychologie (GIO) 50 (2): 141-44.

Schindler, Raoul. 2016. Das lebendige Gefüge der Gruppe. Ausgewählte Schriften. Gießen: Psychosozial-Verlag. 
Schmidt, Johannes, Johannes Brunzel und Dietrich von der Oelsnitz. 2020. „Signale der Macht. Eine Analyse indirekt wahrnehmbarer Machtindikatoren«. Zeitschrift Führung + Organisation 89 (3): 146-51.

Scholl, Wolfgang. 2020. »New Team-Work - eine neue Balance von Gruppe und Führung«. Organisationsberatung, Supervision, Coaching 27 (2): 139-54.

Schrader, Oliver und Lothar Wenzl. 2015. Die Spielregeln der Führung. Erfahrungen und Erkenntnisse aus Unternehmen. Stuttgart: Schäffer-Poeschel.

Schreyögg, Georg und Daniel Geiger. 2016. Organisation. Grundlagen moderner Organisationsgestaltung. 6. Aufl. Wiesbaden: Springer Gabler.

Sichler, Ralph. 2006. Autonomie in der Arbeitswelt. Göttingen: Vandenhoeck \& Ruprecht.

Strauch, Barbara und Annewiek Reijmer. 2018. Soziokratie: Kreisstrukturen als Organisationsprinzip zur Stärkung der Mitverantwortung des Einzelnen. München: Vahlen.

Ulrich, Hans. 1969. »Kompetenz«. In Handwörterbuch der Organisation, hrsg. v. Erwin Grochla, 852-56. Stuttgart: Carl Ernst Poeschel.

Wasserman, Stanley und Katherine Faust. 1994. Social Network Analysis: Methods and Applications. Cambridge: Cambridge University Press.

Watzlawick, Paul, Janet H. Beavin und Don D. Jackson. 2011. Menschliche Kommunikation. Formen, Störungen, Paradoxien. 12. Aufl. Bern: Huber.

Weber, Max. 1972. Wirtschaft und Gesellschaft. 5. Aufl. Tübingen: Mohr Siebeck.

Wegner, Daniel M. 1987. „Transactive Memory: A Contemporary Analysis of the Group Mind«. In Theories of Group Behavior, hrsg. v. Brian Mullen und George R. Goethals, 185-208. New York: Springer.

West, Michael A. 2012. Effective Teamwork. Practical Lessons from Organizational Research. 3. Aufl. Chichester: Wiley-Blackwell.

Wilson, Ernest J. 2008. »Hard Power, Soft Power, Smart Power«. The Annals of the American Academy of Political and Social Science 616: 110-24.

Zaleznik, Abraham. 2009: »The Leadership Gap«. In Change Management, Bd. 1, hrsg. v. Derek S. Pugh und David Mayle, 74-91. Los Angeles: Sage.

\section{Die Autorlnnen}

Michael W. Busch, Priv.-Doz., Dr. rer. pol., wissenschaftlicher Mitarbeiter am Institut für Management \& Leadership Development an der Fachhochschule Wiener Neustadt. Arbeitsschwerpunkte: Grundlagen erfolgreicher Teamarbeit, Führungs- und Organisationsforschung.

Kontakt:michael.busch@fhwn.ac.at

Karin Link, Dr. rer. soc. oec., Leiterin des Instituts für Persönliche Kompetenzentwicklung an der Fachhochschule Wiener Neustadt. Arbeitsschwerpunkte: Leadership, Kompetenzentwicklung, Führungs- und Organisationsforschung.

Kontakt: karin.link@fhwn.ac.at 